$\frac{48}{3-3}-927 \leq(2)$

\section{PPPL-2831}

UC-427
PREPARED FOR THE U.S. DEPARTMENT OF ENERGY, UNDER CONTRACT DE-AC02-76-CHO-3073

PPPL-2831
ATOMIC PHYSICS EFFECTS ON

DISSIPATIVE TOROIDAL DRIFT WAVE STABILITY

BY

M.A. BEER AND T.S. HAHM

February 1992
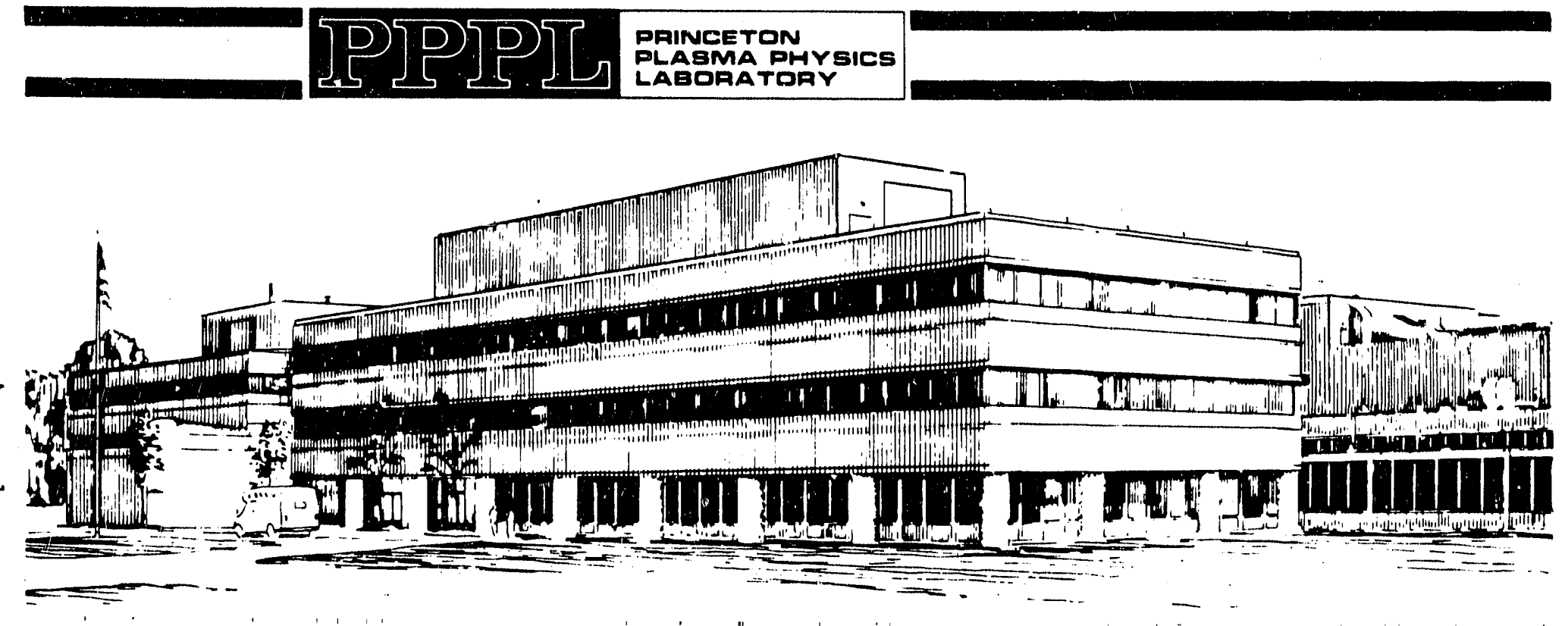


\section{NOTICE}

This report was prepared as an account of work sponsored by an agency of the United States Government. Neither the United States Government nor any agency thereof, nor any of their employees, makes any warranty, express or implied, or assumes any legal liability or responsibility for the accuracy, completeness, or usefulness of any information, apparatus, product, or process disclosed, or represents that its use would not infringe privately owned rights. Reference herein to any specific commercial produce, process, or service by trade name, trademark, manufacturer, or otherwise, does not necessarily constitute or imply its endorsement, rncommendation, or favoring by the United States Government or any agency thereof. The views and opinions of authors expressed herein do not necessarily state or reflect those of the United States Government or any agency thereof.

\section{NOTICE}

This report has been reproduced directly from the best available copy.

Available to DOE and DOE contractors from the:

Office of Scientific and Technical Information P.O. Box 62

Oak Ridge, TN 37831 ;

Prices available from (615) 576-8401.

Available to the public from the:

National Technical Information Service

U.S. Department of Commerce

5285 Port Royal Road

Springfield, Virginia 22161

$703-487-4.650$ 


\title{
Atomic Physics Effects on Dissipative Toroidal Drift Wave Stability
}

\author{
M. A. Beer and T. S. Hahm \\ Princeton Plasma Physics Laboratory, Princeton University \\ Princeton, New Jersey 08543
}

\begin{abstract}
The effects of atomic physics processes such as ionization, charge exchange, and radiation on the linear stability of dissipative drift waves are investigated in toroidal geometry both numerically and analytically. For typical TFTR and TEXT edge parameters, overall linear stability is determined by the competition between the destabilizing influence of ionization and the stabilizing effect due to the electron temperature gradient. An analytical expression for the linear marginal stability condition, $\eta_{e}^{c r i t}$, is derived. The instability is most likely to occur at the extreme edge of tokamaks with a significant ionization source and a steep electron density gradient.
\end{abstract}

PACS numbers: 52.35.Kt, 52.35.Qz, 52.25.Gj, 52.55.Fa 


\section{Introduction}

Tokamak edge plasmas are characterized by large density and potential fluctuations and large particle diffusivity, which typically increases toward the edge as a function of minor radius. ${ }^{1-3}$ Most previous drift wave turbulence models have not been successful in explaining the radial profile of the diffusivity and the large fluctuation levels. ${ }^{4}$ Another widely studied edge turbulence candidate is resistivity gradient driven turbulence ${ }^{5}$ which evolves from the rippling instability ${ }^{6}$ in the linear regime. Although this model predicts larger fluctuation levels and radially increasing particle diffusivities, it suffers from the explicit dependence on the edge current density, which has been called into question by a number of current ramp experiments. ${ }^{7,8}$ However, the radial gradient of the toroidal electric field which develops during the ramp could drive some residual instability. Including realistic effects such as the impurity dynamics ${ }^{9,10}$ and radiation ${ }^{11}$ tends to make the theoretical predictions closer to experimental observations.

Recently, drift wave turbulence has been reconsidered including atomic physics effects with a hope that the aforementioned problems could be remedied. Most of these efforts have been performed in a simplified geometry, with a focus on the nonlinear regime. ${ }^{12,13}$

In this paper, we consider the effects of atornic physics on the 'inear stability of dissipative drift waves in toroidal geometry. It has been shown that in toroidal geometry, the coupling of adjacent poloidal harmonics renders magnetic shear induced damping ineffective. ${ }^{14}$ Therefore, the instability with toroidal mode structure is more likely to be excited than one with Pearlstein-Berk mode structure.

The principal conclusions of this paper include:

i. For typical TFTR and TEXT edge parameters, atomic physics effects are only important 
for long wavelength modes. In order of relative importance: ionization is destabilizing, charge-exchange is stabilizing, and radiation effects are smaller.

ii. It is shown that nonlocal analysis is necessary to properly determine stablility.

iii. For typical parameters, the toroidal mode structure ${ }^{14}$ is maintained in the presence of atomic physics effects. The electron dynamics are nearly adiabatic (Boltzmann-like), rather than hydrodynamic, over most of the width of the eigenmode, although the deviation from adiabaticity is crucial and non-negigible.

iv. Various asymptotic regimes in parameter space are identified and fluctuation characteristics are discussed for each regime. Relations to previous works are elucidated.

The organization of the rest of this paper is as follows. In Sec. II, we present the basic model and obtain the dispersion relation in the local limit. The basic trends atomics physics effects have on stability are indicated by local analysis. We turn to the nonlocal analysis in Sec. III, where we derive the eigenmode equation using the ballooning transformation, and solve it both numerically and analytically. Conclusions and discussion of these results are presented in Sec. IV.

\section{Ii Theoretical Model and Local Analysis}

The basic model for dissipative drift waves can be derived from the Braginskii fluid equations. ${ }^{15}$ To properly include atomic physics effects, we take moments of the Boltzmann equation:

$$
\frac{\partial f}{\partial t}+\mathbf{v} \cdot \nabla_{x} f+\frac{q}{m}\left(\mathbf{E}+\frac{\mathbf{v} \times \mathbf{B}}{c}\right) \cdot \nabla_{v} f=\frac{\delta f}{\delta t} .
$$


obtaining, for the zeroth, first, and second velocity moments:

$$
\begin{aligned}
\frac{\partial n}{\delta^{\prime} t}+\nabla \cdot(n \mathbf{u}) & =\int_{-\infty}^{\infty} d \mathbf{v} \frac{\delta f}{\delta t}, \\
n m \frac{d \mathbf{u}}{d t} & =-\nabla p+q n\left(\mathbf{E}+\frac{\mathbf{u} \times \mathbf{B}}{c}\right)+m \int_{-\infty}^{\infty} d \mathbf{v} \frac{\delta f}{\delta t}(\mathbf{v}-\mathbf{u}), \\
\frac{3}{2} n \frac{d T}{d t} & =-p \nabla \cdot \mathbf{u}-\nabla \cdot \mathbf{q}+\frac{m}{2} \int_{-\infty}^{\infty} d \mathbf{v} \frac{\delta f}{\delta t}(\mathbf{v}-\mathbf{u})^{2}-T \int_{-\infty}^{\infty} d \mathbf{v} \frac{\delta f}{\delta t} .
\end{aligned}
$$

Atomic physics processes and collisions enter these moment equations through the velocity space integrals of

$$
\frac{\delta f}{\delta t}=\left(\frac{\delta f}{\delta t}\right)_{\text {coll }}+\left(\frac{\delta f}{\delta t}\right)_{i o n}+\left(\frac{\delta f}{\delta t}\right)_{c x}+\left(\frac{\delta f}{\delta t}\right)_{\text {rad }}
$$

Therefore, the usual cold ion Braginskii equations are modified in the following way:

$$
\begin{aligned}
\frac{\partial n_{e}}{\partial t}+\nabla \cdot\left(n_{e} \mathbf{v}_{e}\right) & =S_{e} \\
m_{e} n_{e} \frac{d \mathbf{v}_{e}}{d t} & =-\nabla p_{e}-e n_{e}\left(\mathbf{E}+\frac{\mathbf{v}_{e} \times \mathbf{B}}{c}\right)+\mathbf{R}_{e i}+m_{e} S_{e}\left(\mathbf{v} v-\mathbf{v}_{e}\right), \\
\frac{3}{2} n_{e} \frac{d T_{e}}{d t} & =-p_{e} \nabla \cdot \mathbf{v}_{e}-\nabla \cdot \mathbf{q}_{e}+Q_{e i}-P_{r a d}-T_{e} S_{e}+\frac{m_{e}}{2} S_{e}\left(\mathbf{v}_{N}-\mathbf{v}_{e}\right)^{2}, \\
\frac{\partial n_{i}}{\partial t}+\nabla \cdot\left(n_{e} \mathbf{v}_{i}\right) & =S_{i}, \\
m_{i} n_{i} \frac{d \mathbf{v}_{i}}{d t} & =e n_{i}\left(\mathbf{E}+\frac{\mathbf{v}_{i} \times \mathbf{B}}{c}\right)-\mathbf{R}_{e i}+m_{i} D_{i}\left(\mathbf{v}_{N}-\mathbf{v}_{i}\right)+m_{i} S_{i}\left(\mathbf{v}_{N}-\mathbf{v}_{i}\right) .
\end{aligned}
$$

The atomic physics effects are contained in:

$$
\begin{aligned}
S_{e}=S_{i} & =n_{e} n_{N}\langle\sigma v\rangle_{i o n} \\
D_{i} & =n_{i} n_{N}\langle\sigma v\rangle_{c x} \\
P_{\text {rad }} & =n_{e} n_{z} L_{z}\left(T_{e}\right)
\end{aligned}
$$

for ionization, charge exchange, and radiation, respectively. Physically ionization is a source in the electron and ion continuity equations, charge exchange causes drag on the ion parallel velocity, and adiation cools the electrons. Note that while ionization does not affect the fluid 
momentum $(n \mathbf{v})$ or energy $(n T)$, it appears as a drag in the velocity equation, and also enters the temperature equation.

For electrostatic perturbations with $\omega \ll \Omega_{i}$. the perpendicular dynamics are governed by:

$$
\begin{array}{ll}
\mathbf{v}_{\perp_{e}}=\mathbf{v}_{E \times B}+\mathbf{v}_{d e} & \mathbf{v}_{E \times B}=-\frac{c}{B^{2}} \nabla_{\perp} \Phi \times \mathrm{B} \\
\mathbf{v}_{\perp i}=\mathbf{v}_{E \times B}+\mathbf{v}_{p i} & \mathbf{v}_{d e}=-\frac{c}{e B^{2} n_{e}} \mathbf{B} \times \nabla_{\perp} p_{e} \\
& \mathbf{v}_{p i}=-\frac{c}{B \Omega_{i}} \frac{\partial}{\partial t} \nabla_{\perp} \Phi .
\end{array}
$$

Here we have used $m_{i} \gg m_{e}$ and assumed $T_{i} \ll T_{e}$ for simplicity.

In a toroidal system, the divergence of the $\mathrm{E} \times \mathrm{B}$ drift in the ion continuity equation does not vanish, and leads to coupling between different poloidal harmonics. For a large aspect ratio torus with concentric circular flux surfaces, we have $\nabla_{\perp} \cdot \mathbf{v}_{E \times B}=-2 i \omega_{d e}\left(\cos \theta-\frac{i}{k_{\theta}} \sin \theta \cdot \frac{\partial}{\partial r}\right) \frac{e \Phi}{T}$, where $\omega_{d e}=\frac{k_{\theta} \rho_{s} c_{g}}{R}$.

We linearize Eqs. (4)-(8) with $n_{e}=n_{0}+n_{e 1}, n_{i}=n_{0}+n_{i 1}, p_{e}=p_{0}+p_{e 1}, T_{e}=T_{0}+T_{e 1}$, $v_{\|}=v_{\| 1}$, assuming there is no mean flow $\left(v_{\| 0}=0\right)$. The neutral velocity is assumed to be stationary compared to the perturbed ion and electron velocities. We also assume that the wavelengths of the fluctuations are much shorter than the equilibrium gradient scale lengths $\left(L_{n}, L_{T}, \ldots\right)$ but comparable to the ion gyroradius at the electron temperature, $\rho_{s}=\frac{c_{a}}{\Omega_{i}}$. Defining the following dimensionless field quantities,

$$
\tilde{n}_{e}=\frac{n_{e 1}}{n_{0}}, \tilde{n}_{i}=\frac{n_{i 1}}{n_{0}}, \dot{v}_{i}=\frac{v_{\| i}^{\prime}}{c_{s}}, \tilde{v}_{e}=\frac{v_{\| e}}{c_{s}}, \tilde{T}=\frac{T_{e 1}}{T_{0}}, \tilde{\Phi}=\frac{e \Phi}{T_{0}}
$$

and the following atomic process rates,

$$
\begin{aligned}
& \beta_{n}=\frac{\partial S_{e}}{\partial n_{e}}=n_{. v}\langle\sigma v\rangle_{i o n} \\
& \beta_{T}=\frac{T_{0}}{n_{0}} \frac{\partial S_{e}}{\partial T_{e}}=n_{v} T_{0} \frac{\partial\langle\sigma v\rangle_{i o n}}{\partial T_{e}}
\end{aligned}
$$




$$
\begin{aligned}
\gamma_{v} & =\frac{D_{i}}{n_{0}}=n_{N}\langle\sigma v\rangle_{c x} \\
\gamma_{n} & =\frac{2}{3} \frac{1}{T_{0}} \frac{\partial P_{\text {rad }}}{\partial n_{e}}=\frac{2}{3} \frac{n_{z}}{T_{0}} L_{z} \\
\gamma_{T} & =\frac{2}{3} \frac{1}{n_{0}} \frac{\partial P_{\text {rad }}}{\partial T_{e}}=\frac{2}{3} n_{z} \frac{\partial L_{z}}{\partial T_{e}}
\end{aligned}
$$

we have,

$$
\begin{aligned}
\tilde{n}_{e} & =\frac{\omega_{*}}{\omega} \tilde{\Phi}+\frac{k_{\|} c_{s}}{\omega} \tilde{v}_{e}+i \frac{\beta_{n}}{\omega} \tilde{n}_{e}+i \frac{\beta_{T}}{\omega} \tilde{T} \\
0.51 \nu_{e i}\left(\tilde{v}_{i}-\tilde{v}_{e}\right) & =i \frac{k_{\|} v_{t e}^{2}}{c_{s}}\left(\tilde{n}_{e}-\tilde{\Phi}+1.71 \tilde{T}\right)+\beta_{n} \tilde{v}_{e}+i \omega \tilde{v}_{e} \\
\tilde{T} & =\eta_{e} \frac{\omega_{*}}{\omega} \tilde{\Phi}+\frac{2}{3} \frac{k_{\|} c_{s}}{\omega} \tilde{v}_{e}-i \frac{2}{3} 3.16 \frac{k_{\|}^{2} v_{t e}^{2}}{\omega \nu_{e i}} \tilde{T}-\frac{2}{3} 0.71 \frac{k_{\|} c_{s}}{\omega}\left(\tilde{v}_{i}-\tilde{v}_{e}\right) \\
& -i \frac{\gamma_{n}}{\omega} \tilde{n}_{e}-i \frac{\gamma_{T}}{\omega} \tilde{T}-i \frac{2}{3}\left(\frac{\beta_{n}}{\omega} \tilde{n}_{e}+\frac{\beta_{n}}{\omega} \tilde{T}+\frac{\beta_{T}}{\omega} \tilde{T}\right) \\
\tilde{n}_{i} & =\left(\frac{\omega_{*}}{\omega}-2 \frac{\omega_{d e}}{\omega}\left(\cos \theta-\frac{i}{k_{\theta}} \sin \theta \frac{\partial}{\partial r}\right)+\rho_{s}^{2}\left(\frac{\partial^{2}}{\partial r^{2}}-k_{\theta}^{2}\right)\right) \tilde{\Phi}+\frac{k_{\|} c_{s}}{\omega} \tilde{v}_{i} \\
\tilde{n}_{e}+i \frac{\beta_{T}}{\omega} \tilde{T} & \\
\tilde{v}_{i} & =\frac{k_{\|} c_{s}}{\omega}\left(\tilde{n}_{e}+\tilde{T}\right)-i \frac{\gamma_{v}}{\omega} \tilde{v}_{i}-i \frac{\beta_{n}}{\omega} \tilde{v}_{i}
\end{aligned}
$$

where the numerical factors come from the Braginskii coefficients $\left(\eta_{\|}=0.51 \frac{m_{e} \nu_{e 1}}{n e^{2}}, \kappa_{\|}=3.16 \frac{n T}{m_{\varepsilon} \nu_{\theta 1}}\right.$, $\alpha=1.71$ ) and $\eta_{e}=\frac{L_{n}}{L_{T}}, \omega_{*}=\frac{k_{\theta} \rho_{s} c_{s}}{L_{n}}$. We note that density and temperature fluctuations affect the ionization source $\left(\beta_{n}, \beta_{T}\right)$, and the radiated power $\left(\gamma_{n}, \gamma_{T}\right)$.

Before presenting the solution of the eigenmode cquation (in Sec. III), which is necessary to accurately determine the stability of this system, we begin by examining the local dispersion relation. For the purposes of this discussion, we treat the $\omega_{d e}$ term as a constant, evaluated at $\theta=0$ (bad curvature side), and treat $-\frac{\partial^{2}}{\partial r^{2}}-k_{\theta}^{2}$ as a constant, $k_{\perp}^{2}$. In this local limit,

$$
\tilde{n}_{i}=\left(\frac{\omega_{*}}{\omega}-2 \frac{\omega_{d e}}{\omega}-k_{\perp}^{2} \rho_{s}^{2}\right) \tilde{\Phi}+\frac{k_{\|} c_{s}}{\omega} \tilde{v}_{i}+i \frac{\beta_{n}}{\omega} \tilde{n}_{e}+i \frac{\beta_{T}}{\omega} \tilde{T}
$$


Using quasineutrality and Eqs. (9)-(11) and (13), we can obtain the local dispersion relation.

Two dimensionless parameters play crucial roles in characterizing the properties of the fluctuations: $\frac{k_{\|}^{2} v_{t e}^{2}}{\omega \nu_{e i}}$ and $\frac{k_{\|}^{2} c_{s}^{2}}{\omega^{2}}$. The first, $\frac{k_{\|}^{2} v_{t e}^{2}}{\nu_{e i}}$ comes from the $\kappa_{\|} \nabla_{\|}^{2} T_{e}$ term in $\nabla \cdot \mathrm{q}_{e}$, and measures electron thermal conduction along $B$. Thus the parameter $\frac{k_{\|}^{2} v_{l e}^{2}}{\omega \nu_{e i}}$ compares the parallel electron thermal conduction rate to the mode frequency. The second, $\frac{k_{\|} c_{s}}{\omega}$ is the ratio of the sound wave propagation rate along $\mathbf{B}$ to the mode frequency. When $\frac{k_{1}^{2} \nu_{t e}^{2}}{\omega \nu_{e i}} \gg 1$, electron thermal conduction is strong enough to smooth temperature fluctuations along $\mathbf{B}$, so $\tilde{T}_{e} \rightarrow 0$. This is known as the adiabatic limit, and electrons have a Boltzmann-like distribution. When $\frac{k_{11}^{2} c_{s}^{2}}{\omega^{2}} \gg 1$, sound waves smooth pressure perturbations aiong $\mathrm{B}$, so $\tilde{p}_{e} \rightarrow 0$. This will be called the pressure balance limit in this paper.

As will be shown in Sec. III using the nonlocal mode structure, the adiabatic regime is relevant for typical tokamak edge parameters. We discuss several other limits to relate this work to previous studies.

i). In the adiabatic regime, $\frac{k_{\|}^{2} v_{t e}^{2}}{\omega \nu_{e l}} \gg 1, \frac{k_{1}^{2} c_{s}^{2}}{\omega^{2}}$ :

$$
\tilde{n}_{e} \simeq \tilde{\Phi}, \quad \tilde{T} \simeq 0, \quad \tilde{v}_{i} \simeq \frac{k_{\|} c_{s}}{\omega+i \gamma_{v}+i \beta_{n}} \tilde{\Phi}
$$

Quasi-neutrality gives the following dispersion relation to the leading order in $\frac{\omega \nu_{\text {ei }}}{k_{i \|}^{2} v_{t e}^{t}}$ :

$$
\omega^{2}(1+b)-\omega\left(\omega_{*}-2 \omega_{d e}+i \beta_{n}-i(1+b)\left(\gamma_{v}+\beta_{n}\right)\right)-k_{\|}^{2} c_{s}^{2}-i\left(\gamma_{v}+\beta_{n}\right)\left(\omega_{*}-2 \omega_{d e}+i \beta_{n}\right)=0
$$

where $b=k_{\perp}^{2} \rho_{s}^{2}$. When $k_{\|} c_{s}, \gamma_{v}, \beta_{n} \ll \omega$, the electron root further reduces to:

$$
\omega_{0} \simeq \frac{\omega_{*}-2 \omega_{d e}+i \beta_{n}}{1+b}+\frac{k_{\|}^{2} c_{s}^{2}}{\left(\omega_{*}-2 \omega_{d e}\right)^{2}}\left[\omega_{*}-2 \omega_{d e}-i \beta_{n}-i\left(\beta_{n}+\gamma_{v}\right)(1+b)\right] \text {. }
$$

In the local approximation, the destabilizing effect of ionization $\left(\beta_{n}\right)$ comes from the inverse dissipation in the ion continuity equation. The stabilizing influence due to the ionization 
and charg|z-exchange drag on the parallel ion velocity is smaller roughly by a factor of $\frac{k_{11}^{2} c_{s}^{2}}{\omega^{2}}$. When spatially dependent $k_{\|}$is actually taken into account, a nonlocal analysis in Sec. III shows that the overall effect of ionization is destabilizing. Therefore, the ionization term in the ion velocity equation, which is often neglected in the previous studies, seems to be important only when $\frac{k_{l}^{2} c_{s}^{2}}{\omega^{s}} \gtrsim 1$. We also note that the real frequency is shifted below $\omega_{*}$ by $\omega_{\text {de }}$ and finite Larmor radius effects. Because $\tilde{T}$ is small in this regime, radiation effects are subdominant.

Collisional effects appear as a first order $\left(\sim \frac{\omega \nu_{e i}}{k_{\|}^{2} v_{t e}^{2}}\right)$ correction to $\omega_{0}$ in Eq. (16);

$$
\omega \simeq \omega_{0}+i \frac{0.812}{(1+b)^{2}} \frac{\omega_{m}^{2} \nu_{e i}}{k_{\|}^{2} v_{t e}^{2}}\left[1.77\left(1-\frac{\omega_{0}}{\omega_{*}}\right)-\eta_{e}\right] \text {. }
$$

Collisional effects are destabilizing when the down-shift of $\omega$ overcomes the stabilizing effects of $\eta_{e}$, i.e., $\eta_{e}<1.77\left(1-\frac{\omega}{\omega_{*}}\right)$. When the spatial dependence of $k_{\|}$is treated nonlocally in the next section, this scaling with $\nu_{e i}$ changes to $\nu_{e i}^{\frac{1}{2}}$, increasing the relative importance of collisional effects. Radiation effects also enter at this order in $\frac{\omega \nu_{e+}}{k_{\| l}^{2} v_{t e}^{2}}$, but only shift the real frequency, and do not affect stability directly.

ii). In the hydrodynamic regime, $\frac{k_{\|}^{2} v_{t e}^{2}}{\omega \nu_{e 1}}, \frac{k_{j !}^{2} c_{s}^{2}}{\omega^{2}} \ll 1$ :

$$
\tilde{n}_{e} \simeq \frac{\omega_{*}}{\omega} \tilde{\Phi}, \quad \tilde{T}_{e} \simeq \eta_{e} \frac{\omega_{*}}{\omega} \tilde{\Phi}
$$

Here the dynamics along $B$ is insignificant, and the electron density and temperature are mainly $\mathbf{E} \times \mathbf{B}$ convected. Note that if $\eta_{e} \gg 1$, we recover the limit considered by Ware, et al. ${ }^{12}$ where $\omega \sim \eta \omega_{*}=\omega_{* T}$ and $\tilde{T} \sim \tilde{\Phi} \gg \tilde{n}_{e}$ In this limit, radiation effects could be significant. 
iii). In the pressure balance regime, $\frac{k_{\|}^{2} c_{s}^{2}}{\omega^{2}} \gg 1, \frac{k_{\|}^{2} v_{l e}^{2}}{\omega \nu_{e i}}$ :

$$
\tilde{n}_{e} \simeq-\tilde{T} \simeq\left[\frac{\frac{\omega_{e}}{\omega}\left(\frac{2}{3}-\eta_{e}\right)+i 1.82 \frac{k_{\|}^{2} v_{t e}^{2}}{\omega \nu_{e i}}}{\frac{5}{3}+i 5.51 \frac{k_{1}^{2} v_{t e}^{2}}{\omega \nu_{e i}}}\right] \tilde{\Phi}
$$

This limit is similar to the one investigated by Drake, et al. ${ }^{16}$ for the case $\tilde{\mathfrak{J}} \simeq 0$. In contrast to the near adiabatic regime, the pressure balance regime permits large electron temperature fluctuations, and consequently, radiation could become an important destabilizing effect.

\section{Nonlocal Analysis}

We derive the linear eigenmode equation using the ballooning formalism. ${ }^{17-19}$ This procedure reduces the two dimensional problem in $(r, \theta)$ to a one dimensional eigenmode equation in the ballooning coordinate $(\eta)$, which can be regarded as the coordinate along the field lines. The simplifying assumption is that for large mode numbers $m$, different poloidal harmonics have nearly identical structures centered at each rational surface.

Using quasineutrality, Eqs. (12) and (13) become:

$$
\begin{gathered}
\left(1-\frac{k_{\|}^{2} c_{s}^{2}}{\omega^{2}}-\frac{\omega_{*}}{\omega}+2 \frac{\omega_{d e}}{\omega}\left(\cos \theta-\frac{i}{k_{\theta}} \sin \theta \frac{\partial}{\partial r}\right)-\rho_{s}^{2}\left(\frac{\partial^{2}}{\partial r^{2}}-k_{\theta}^{2}\right)\right) \tilde{\Phi}= \\
-\left(1-\frac{k_{\|}^{2} c_{s}^{2}}{\omega^{2}}\right) \delta \tilde{n}_{e}+\left(i \frac{\beta_{n}}{\omega}-\frac{i\left(\gamma_{v}+\beta_{n}\right) k_{\|}^{2} c_{s}^{2}}{\omega^{2}\left(\omega+i \gamma_{v}+i \beta_{n}\right)}\right) \tilde{n}_{e}+\left(i \frac{\beta_{T}}{\omega}+\frac{k_{\|}^{2} c_{s}^{2}}{\omega\left(\omega+i \gamma_{v}+i \beta_{n}\right)}\right) \tilde{T} .
\end{gathered}
$$

Here we have broken the perturbed electron density into its adiabatic and non-adiabatic parts: $\tilde{n}_{e}=\tilde{\Phi}+\delta \tilde{n}_{e}$. The right-hand side of Eq. (19) contains the non-adiabatic electron response and the atomic physics effects. Because the electrons are nearly adiabatic and atomic physics effects are small for typical TEXT and TFTR parameters considered here, we treat the right hand side of Eq. (19) perturbatively. 
We use the usual $(r, \theta, \xi)$ coordinates, corresponding to the minor radial, poloidal, and toroidal directions. In a large aspect-ratio axisymmetric torus, the perturbed potential can be expressed as:

$$
\tilde{\Phi}(r, \theta, \xi)=\sum_{j} \hat{\Phi} \cdot(s) \exp \left[i\left(m_{0} \theta+j \theta-n \xi-\omega t\right)\right],
$$

where $|j| \ll m_{0}, s=\frac{\left(r-r_{0}\right)}{\Delta r_{s}}, r_{0}$ is the reference rational surface $m_{0}=n q\left(r_{0}\right), \Delta r_{s}=\frac{1}{k_{\theta} \dot{s}}, k_{\theta}=\frac{m_{0}}{r_{0}}$, and $\hat{s}=\frac{r q 1}{q}$ at $r=r_{0}$. Ignoring for the moment the right-hand side, Eq. (19) is:

$$
\left[1-\frac{1}{\Omega}-\frac{(s-j)^{2}}{\eta_{s}^{2} \Omega^{2}}-b_{\theta}\left(\hat{s}^{2} \frac{\partial^{2}}{\partial s^{2}}-1\right)\right] \hat{\Phi}_{j}+\frac{\epsilon_{n}}{\Omega}\left[\hat{\Phi}_{j-1}+\hat{\Phi}_{j+1}-\hat{s} \frac{\partial}{\partial s}\left(\hat{\Phi}_{j-1}-\hat{\Phi}_{j+1}\right)\right]=0
$$

where we have used $k_{\|}=\frac{(s-j)}{q R}, b_{\theta}=k_{\ell}^{2} \rho_{s}^{2}, \Omega=\frac{\omega}{\omega_{0}}, \eta_{s}=\frac{q b_{\theta}^{\frac{1}{2}}}{\epsilon_{n}}, \epsilon_{n}=\frac{L_{n}}{R}$. Using the ballooning mode formalism, for $\left|m_{0}\right| \sim|n| \gg 1, \hat{\Phi}_{j}(s)=\Phi(z)$, and $\hat{\Phi}_{j \pm 1}(s)=\Phi(z \mp 1)$, with $z=s-j$. In this approximation, the eigenmodes are composed of identical structures centered at each rational surface. The eigenmode equation is:

$$
\left[1-\frac{1}{\Omega}-\frac{z^{2}}{\eta_{s}^{2} \Omega^{2}}-b_{\theta}\left(\hat{s}^{2} \frac{d^{2}}{d z^{2}}-1\right)\right] \Phi(z)+\frac{\epsilon_{n}}{\Omega}\left[\Phi(z+1)+\Phi(z-1)-\hat{s} \frac{d}{d z}[\Phi(z+1)-\Phi(z-1)]\right]=C
$$

We now Fourier transform this equation:

$$
\Phi(\eta)=\int_{-\infty}^{\infty} d z e^{i \eta z} \Phi(z)
$$

The eigenmode equation is now a one-dimensional differential equation in the ballooning coordinate, $\eta:^{14}$

$$
\left[\frac{d^{2}}{d \eta^{2}}+Q(\eta, \Omega)\right] \Phi(\eta)=0
$$

where

$$
Q(\eta, \Omega)=\eta_{s}^{2} \Omega^{2}\left[1-\frac{1}{\Omega}+b_{\theta}\left(1+\dot{s}^{2} \eta^{2}\right)+\frac{2 \epsilon_{n}}{\Omega}(\cos \eta+s \eta \sin \eta)\right] .
$$


The $\frac{d^{2}}{d \eta^{2}}$ term is the ion sound wave contibution. In the expression for $Q, 1$ is the Boltzmann electron response, $-\frac{1}{\Omega}$ is the $\mathbf{E} \times \mathbf{B}$ convection of the ion density, $\hat{s}^{2} \eta^{2}$ is the ion polarization drift, and $\frac{2 \epsilon_{n}}{\Omega}(\cos \eta+\hat{s} \eta \sin \eta)$ is the toroidal coupling term. We repeat this procedure including the right-hand side of Eq. (19), and obtain:

$$
\begin{gathered}
{\left[\frac{d^{2}}{d \eta^{2}}+Q(\eta, \Omega)+\delta Q(\eta, \Omega)\right] \Phi(\eta)=0} \\
\delta Q(\eta, \Omega)=\frac{\eta_{s}^{2} \Omega^{2}}{\Phi(\eta)} \int_{-\infty}^{\infty} d z e^{i \eta z} \Phi(z)\left[\left(1-\frac{z^{2}}{\eta_{s}^{2} \Omega^{2}}\right) \delta \tilde{n}_{e}\right. \\
\left.-i\left(\frac{\frac{\beta_{n}}{\omega}}{\Omega}-\frac{\frac{\gamma_{v}}{\omega_{*}}+\frac{\beta_{n}}{\Omega+i \frac{\nu_{v}}{\omega_{*}}}+i \frac{\beta_{n}}{\omega_{*}}}{\eta_{s}^{2} \Omega^{2}}\right) \tilde{n}_{e}-\left(\frac{i \frac{\beta_{T}}{\omega_{*}}}{\Omega}+\frac{\Omega}{\Omega+i \frac{\gamma_{v}}{\omega_{*}}+i \frac{\beta_{n}}{\omega_{*}}} \frac{z^{2}}{\eta_{s}^{2} \Omega^{2}}\right) \tilde{T}\right]
\end{gathered}
$$

where $\Phi(z)=\frac{1}{2 \pi} \int_{-\infty}^{\infty} d z e^{-i \eta z} \Phi(\eta)$.

We solve this equation as follows. First, we find the lowest order eigenfunction using adiabatic electrons $(\delta Q=0)$. Then using this lowest order eigenfunction, we evaluate $\delta Q(\eta)$ in Eq. (25b). We find that $\delta Q(\eta)$ is constant over the eigenmode $\Phi(\eta)$ to a very good approximation. For small and constant $\delta Q$, the effect on stability is simply $\operatorname{Im}\left(\omega / \omega_{*}\right) \simeq-\operatorname{Im}(\delta Q) / \eta_{s}^{2} \Omega^{2}$. As far as linear anaylsis is concerned, TFTR and TEXT parameters justify the perturbative treatument, since $\gamma \ll \omega_{r}$, as will be shown. Equation (24) is the eigenmode equation of Ref. 14, and is obtained from our equations neglecting the non-adiabatic electron response and atomic physics. We solve Eq. (24) numerically using an interactive WKB shooting code. ${ }^{20}$ We are interested in the most unstable modes for this system, which are the neutrally stable toroidicity induced eigenmodes. Figures 1 and 2 show numerical solutions to this equation for typical tokamak parameters, for an extremely long wavelength eigenmode, $k_{\theta} \rho_{s}=0.02$ (Fig. 1), and for a shorter wavelength eigenmode, $k_{\theta} \rho_{s}=0.1$ (Fig. 2). The effective potential, $-Q(\eta)$, is shown in (a). The 
toroidal coupling terms create the local potential well which makes the magnetic shear induced damping ineffective, thus making these modes neutrally stable. The eigenmode structure in the ballooning coordinate, $\Phi(\eta)$, is shown in (b). The eigenmode structure in the radial coordinate, $\Phi(z)$, obtained by Fourier tranforming $\Phi(\eta)$, is shown in (c). The eigenfrequencies for these two cases are $\Omega=0.82$ for $k_{\theta} \rho_{s}=0.02$ and $\Omega=0.48$ for $k_{\theta} \rho_{s}=0.1$.

To proceed, we solve Eqs. (9)-(11) and (13) for $\tilde{n}_{e}, \delta \tilde{n}_{e}$, and $\tilde{T}$ in terms of $\tilde{\Phi}$, and obtain:

$$
\begin{gathered}
\tilde{n}_{e}=\frac{a e+b f}{a d-c f} \tilde{\Phi} \\
\tilde{T}=\frac{b d+c e}{a d-c f} \tilde{\Phi} \\
\delta \tilde{n}_{e}=\frac{a(e-d)+(b+c) f}{a d-c f} \tilde{\Phi}
\end{gathered}
$$

where:

$$
\begin{aligned}
& a=i \frac{2}{3}\left(3.16+\frac{(1.71)^{2}}{0.51}\right) \frac{x^{2}}{\bar{\nu} \Omega}-\frac{2}{3} \frac{x^{2}}{\Omega\left(\Omega+i \frac{\gamma_{v}}{\omega_{\bullet}}+i \frac{\beta_{n}}{\omega_{\bullet}}\right)}+1+i\left(\frac{\gamma_{T}}{\omega}+\frac{2}{3} \frac{\beta_{n}}{\omega}+\frac{2}{3} \frac{\beta_{T}}{\omega}\right) \\
& b=i \frac{2}{3} \frac{1.71}{0.51} \frac{x^{2}}{\bar{\nu} \Omega}+\frac{\eta_{e}}{\Omega} \\
& c=-i \frac{2}{3} \frac{1.71}{0.51} \frac{x^{2}}{\bar{\nu} \Omega}+\frac{2}{3} \frac{x^{2}}{\Omega\left(\Omega+i \frac{\gamma_{v}}{\omega_{*}}+i \frac{\beta_{n}}{\omega_{\bullet}}\right)}-i\left(\frac{\gamma_{n}}{\omega}+\frac{2}{3} \frac{\beta_{n}}{\omega}\right) \\
& d=i \frac{x^{2}}{0.51 \bar{\nu} \Omega}-\frac{x^{2}}{\Omega\left(\Omega+i \frac{\gamma_{v}}{\omega_{\bullet}}+i \frac{\beta_{n}}{\omega *}\right)}+1-i \frac{\beta_{n}}{\omega} \\
& e=i \frac{x^{2}}{0.51 \bar{\nu} \Omega}+\frac{1}{\Omega} \\
& f=-i \frac{1.71}{0.51} \frac{x^{2}}{\bar{\nu} \Omega}+\frac{x^{2}}{\Omega\left(\Omega+i \frac{\gamma_{v}}{\omega_{\bullet}}+i \frac{\beta_{n}}{\omega_{\bullet}}\right)}+i \frac{\beta_{T}}{\omega} .
\end{aligned}
$$

We have normalized $z=x \eta_{s}$, and $\bar{\nu}=\frac{\nu_{\omega} m_{s}}{\omega_{m_{1}} m_{1}}$. These terms in $\delta Q$ are ratios of fourth order polynomials in $z$, and do not Fourier transform nicely into ballooning space as the $z^{2}$ and $\frac{d^{2}}{d z^{2}}$ terms do in the unperturbed eigenmode equation, so we evaluate them in configuration space, and then transform to ballooning space. 
Typical edge parameters for TFTR and TEXT are shown in Table I. $\gamma_{n}$ is calculated using experimentally measured $P_{\text {rad }}$. To estimate $\gamma_{T}$, we use coronal equilibrium cooling rates, ${ }^{21}$ normalized to match $P_{\text {rad }}$. Since the actual $L_{z}\left(T_{e}\right)$ will be smoother than the coronal model, this is an upper bound on $\gamma_{T}$. All atomic physics rates are much smaller than $\omega$. for $m$ values of interest. Using the lowest order eigenfunctions, we car now evaluate $\frac{k_{1}^{2} v_{\text {te }}^{2}}{\omega \nu_{e l}}$ and $\frac{k_{1}^{2} c_{g}^{2}}{\omega^{2}}$, and identify the parameter regime for these fluctuations. For the toroidicity induced modes, $k_{\|} \sim \frac{\Delta x}{L_{s}} k_{\theta}$, where $\Delta x \sim \frac{1}{k_{\theta} \hat{s}}$ and $L_{s}=\frac{q R}{\dot{s}}$, so

$$
\frac{k_{\|}^{2} c_{s}^{2}}{\omega^{2}} \sim\left(\frac{L_{n}}{q R}\right)^{2}\left(\frac{\omega_{*}}{\omega}\right)^{2} \frac{1}{\left(k_{\theta} \rho_{s}\right)^{2}} .
$$

In the TFTR and TEXT edge, this quantity varies from $10^{-3}$ for short wavelengths $\left(k_{\theta} \rho_{s}=1\right)$ to 1 for longer wavelengths $\left(k_{\theta} \rho_{s}=0.05\right)$. Therefore, we have $\frac{k_{1}^{2} c_{s}^{2}}{w^{2}} \lesssim 1$ tur parameters of interest, and pressure balance is not likely to be achieved, even for rather low $k_{\theta} \rho_{\mathbf{s}}(m \sim 50)$.

More importantly, since

$$
\frac{k_{\|}^{2} v_{t e}^{2}}{\omega \nu_{e i}}=\frac{1}{\bar{\nu}} \frac{k_{\|}^{2} c_{s}^{2}}{\omega^{2}} \frac{\omega}{\omega_{*}}
$$

and typically $\bar{\nu} \ll 1$, even for rather low $k_{\theta} \rho_{s}$, we have $\frac{k_{\|}^{2} v_{t e}^{2}}{\omega \nu_{e i}} \gg 1 \gtrsim \frac{k_{1}^{2} c_{s}^{2}}{\omega^{2}}$. In summary, these fluctuations are typically in the adiabatic regime.

Because of the spatial dependence of $k_{\|}$, the eleccrons are hydrodynamic only within a narrow region near the rational surface, and adiabatic outside this region, as shown in Fig. 3. For illustration, Fig. 4 shows the case $\bar{\nu} \gg 1$, where the electrons are hydrodynamic near the rational surface, and pressure balance is enforced outside this region. The typical parameter regimes for toroidal drift waves in TFTR and TEXT are shown in Fig. 5.

We can now evaluate the integral for $\delta Q$ using the lowest order eigenfunctions $\Phi(z)$, as shown in Figs. $1(\mathrm{c})$ and $2(\mathrm{c})$, for example. Since $\bar{\nu} \ll 1$, the electrons are non-adiabatic only within a narrow region near the rational surface, and the integrand for the perturbed potential $\delta Q$ looks 
like a delta function in $z$. When transformed into ballooning space, $\delta Q(\eta)$ is roughly constant over the width of the eigenmode, as shown for these two cases in Figs. 6 and 7 .

If the atomic physics rates are $\eta$-dependent due to specific experimental situations such as the limiter configuration and gas puff location, the imaginary (dissipative) part of $\delta Q$ would be n-dependent and could induce toroidal coupling. ${ }^{22}$ For the parameters considered in this paper, the toroidal coupling in the real (reactive) part of $Q$ caused by ion drifts is a larger effect since $\omega_{\text {de }} \gg \gamma$

In the limit $\bar{\nu} \ll 1$, we can evaluate $\delta Q$ analytically without knowing the detailed eigenmode structure, keeping terms up to $O\left(\bar{\nu}^{\frac{1}{2}}\right)$. The lowest order piece of the $\delta \tilde{n}_{e}$ term in Eq. (25b) contributes

$$
\begin{aligned}
& \delta Q=\eta_{s}^{3} \bar{\nu}^{\frac{1}{2}} \Omega^{\frac{3}{2}} \frac{\sqrt{\pi}}{2}(1-i) \quad\left[2.51(1-\Omega)-1.06 \eta_{e}\right] \\
& +\eta_{s}^{3} \bar{\nu}^{\frac{1}{2}} \Omega^{\frac{1}{2}} \frac{\sqrt{\pi}}{2}(1+i)\left[\frac{\gamma_{T}}{\omega_{e}}\left(0.24 \Omega+0.33(1-\Omega)+0.19 \eta_{e}\right)\right. \\
& +\frac{\gamma_{n}}{\omega_{e}}\left(1.13 \Omega+0.49(1-\Omega)-0.16 \eta_{e}\right) \\
& \left.+\underset{\omega_{0}}{\dot{\beta}_{\dot{T}}}\left(0.35 \Omega+0.02(1-\Omega)+0.87 \eta_{e}\right)\right] \text {. }
\end{aligned}
$$

For the second term in Eq. (25b), $\tilde{n}_{e}=\tilde{\Phi}$ to lowest order. The $\beta_{n}$ term is trivial to evaluate, but for the $\gamma_{v}$ term we approximate $\int_{-\infty}^{\infty} z^{2} \Phi(z) d z \simeq \frac{2}{\pi^{2}}$. The dominant contribution is:

$$
\delta Q=-i \eta_{s}^{2} \Omega^{2} \frac{\beta_{n}}{\omega}+i \frac{2}{\pi^{2}}\left(\frac{\gamma_{v}}{\omega}+\frac{\beta_{n}}{\omega}\right)
$$

The lowest order contribution from the $\tilde{T}$ term is proportional to $\frac{\beta}{\omega} T$ :

$$
\delta Q=\eta_{s}^{3} \frac{1}{2} \Omega^{\frac{1}{2}} \frac{\sqrt{\pi}}{2}(1+i) \frac{\beta_{T}}{\omega_{*}}\left(0.35 \Omega+0.16(1-\Omega)-0.14 \eta_{e}\right) .
$$

Combining these terms, the growth rate is:

$$
\begin{aligned}
\frac{\gamma}{\omega_{*}}= & \frac{\beta_{n}}{\omega}-\frac{2}{\pi^{2} \eta_{s}^{2} \Omega^{2}}\left(\frac{\gamma_{v}}{\omega}+\frac{\beta_{n}}{\omega}\right)+\frac{\sqrt{\pi}}{2} \eta_{s}\left(\frac{\bar{\nu}}{\Omega}\right)^{\frac{1}{2}}\left[2.51(1-\Omega)-1.06 \eta_{e}\right] \\
& -\frac{\sqrt{\pi}}{2} \eta_{s}\left(\frac{\bar{\nu}}{\Omega}\right)^{\frac{1}{2}}\left[\frac{\gamma_{T}}{\omega}\left(0.24 \Omega+0.33(1-\Omega)+0.19 \eta_{e}\right)\right.
\end{aligned}
$$




$$
\begin{aligned}
& +\frac{\gamma_{n}}{\omega}\left(1.13 \Omega+0.49(1-\Omega)-0.16 \eta_{e}\right) \\
& \left.+\frac{\beta_{T}}{\omega}\left(0.69 \Omega+0.17(1-\Omega)+0.74 \eta_{e}\right)\right] .
\end{aligned}
$$

The basic trends of the local analysis remain, with some important modifications. The desta. bilization from the ionization term in the ion continuity equation is reduced by charge-exchange and ionization drag on the ion velocity, but the stabilizing term is smaller by a factor of $\frac{2}{\pi^{2} \eta_{3}^{2} \Omega^{2}}$, which depends on the wavelength $\left(\eta_{s} \sim k_{\theta} \rho_{s}\right)$. As $k_{\theta} \rho_{s}$ gets smaller, the stabilizing term becomes competitive. In the third term, the collisional effects scale like $\frac{1}{2}$ instead of $\bar{\nu}$ predicted from local theory. Therefore, this term is not negligible compared to $\bar{\nu}$-independent terms. The radiation effects are very weak, since $\bar{\nu}, \gamma_{n}$, and $\gamma_{T}$ are all small.

Figures 8(a)-(g) compare the numerical and analytical growth rates as $\bar{\nu}, \eta_{e}, \beta_{n}, \beta_{T}, \gamma_{\nu}, \gamma_{n}$, and $\gamma_{T}$ are varied, using the parameters in Fig. 1.

Typically, the frowth rate is determined by the competition between ionization and collisions, the latter is stabilizing for large $\eta_{e}$, and overall stability is achieved when:

$$
\eta_{e}>\eta_{\text {crit }}=2.37(1-\Omega)+\frac{2}{\sqrt{\pi}} \frac{\beta_{n}}{\omega_{*}}-\frac{1}{(\bar{\nu} \Omega)^{\frac{1}{2}}} \frac{1}{\eta_{s}}
$$

For the cases considered in Table I, collisional stabilization is stronger than the destabilization from ionization, rendering these modes stable. However, at the extreme edge of tokamaks, steeper density gradients and higher ionization rates may make these modes unstable.

\section{Conclusions and Discussion}

This paper has focused on the effects of various atomic physics processes on dissipative drift wave stability in toroidal geometry. The principal conclusions of this paper are as follows. 
i). For typical parameters of the TFTR and TEXT edge, the electron dynamics are nearly adiabatic.

ii). The deviation from the adiabatic response which is required for instability comes from ionization, collisions, charge exchange, and radiation, roughly in order of importance.

iii). For an instability, the inverse dissipation from ionization or from the downshift of the real fi equency below $\omega_{* e}$ should overcome the stabilizing effects due to the electron temperature gradient, quantified by $\eta_{e}$. Therefore, for long wavelength modes, an instability is likely to occur at the extreme edge in minor radius with significant ionization source and rather steep electron density gradient (low $\left.\eta_{e}\right)$.

iv). Ionization acts as an inverse dissipation in the density continuity equation, but also appears as an effective drag in the ion velocity equation. This effect, although ignored in previous studies, ${ }^{13}$ can quantitatively affect stability if $\frac{k_{\|}^{2} c_{s}^{2}}{\omega^{2}} \gtrsim 1$.

v). The magnitudes of two dimensionless parameters, $\frac{k_{\|}^{2} v_{l e}^{2}}{\omega_{\nu_{e l}}}$ and $\frac{k_{\|}^{2} c_{0}^{2}}{w^{2}}$ determine the asymptotic regimes in parameter space and the characteristics of the fluctuations. Relevances of the previous studies ${ }^{11,12,16}$ on radiation-driven edge turbulence models are discussed for certain regimes.

The detailed linear analyses and results presented in this paper indicate that the edge drift instability has a growth rate which is smaller than the real frequency for $m>10$. However, we would like to point our that this does not necessarily imply small fluctuation levels at saturation. Since the longer wavelength fluctuations (with $k_{\perp} \rho_{s} \ll 1$ ) are more likely to be destabilized by the ionization, the weak turbulence analysis based upon nonlinear wave-particle interactions 
(including ion Compton scattering) is not likely to be justified. In the strong turbulence regime, the Hasegawa-Mima nonlinearity ${ }^{23}$ is also negligible because it relies on the nonlinear polarization drift. Therefore, the nonlinear saturation could occur through a nonlinear process which is insensitive to the strength of finite Larmor radius effects. One possibility would be the dissipative

correction to $\mathbf{E} \times \mathbf{B}$ convection. ${ }^{24}$ Since this nonlinearity is also weak, it is possible to have a large fluctuation level at saturation. ${ }^{13}$

\section{Acknowledgments}

We would like to thank G. W. Hammett for pointing out important modifications to the Braginskii equations, and R. Budny and W. L. Rowan for providing the experimental data. We also thank P. H. Diamond for useful sugges ons.

This work was supported by a Nativnal Science Foundation Fellowship and by the United States Department of Energy contract No. DE-AC02-76-CHO-3703.

\section{References}

1 S. J. Zweben and R. W. Gould, Nucl. Fusion 23, 1625 (1983).

${ }^{2}$ Ch. P. Ritz, R. D. Bengston, S. J. Levinson, and E. J. Powers, Phys. Fluids 27, 2956 (1984).

3 A. J. Wootton, B. A. Carreras, H. Matsumoto, K. McGuire, W. A. Peebles, Ch. P. Ritz, P. W. Terry, and S. J. Zweben, Phys. Fluids B 2, 2879 (1990).

4 P. W. Terry and P. H. Diamond, Phys. Fluids 28, 1419 (1985).

5 L. Garcia, P. H. Diamond, B. A. Carreras, and J, D. Callen, Phys. Fluids 28, 2147 (1985). 
6 H. P. Furth, J. Killeen, and M. N. Rosenbluth, Phys. Fluids 6, (1963).

7 K. W. Gentle, R. D. Bengston, R. Bravenec, D. L. Brower, W. L. Hodge, T. P. Kochanski, N. C. Luhmann, D. Patterson, W. S. Peebles, and P. E. Phillips, Plasma Phys. 26, 1407 (1984).

8 M. C. Zarnstorff, R. J. Goldston, E. Fredrickson, C. Bush, B. Grek, T. S. Hahm, D. Johnson, D. Mansfield, D. McCune, H. Park, A. Ramsey, J. Schivell, and G. Taylor, in Proceedings of Annual Controlled Fusion Theory Conference (San Antonio, Texas, 1989).

9 P. H. Rutherford, in Physics of Plasma Close to Thermonuclear Conditions, edited by B. Coppi, et. al. (Pergamon, New York, 1981), Vil. I, p. 143.

10 T. S. Hahm, P. H. Diamond, P. W. Terry, L. Garcia, and B. A. Carreras, Phys, Fluids 30, $1452(1987)$.

11 D. R. Thayer and P. H. Diamond, Phys. Fluids 30, 3724 (1987).

12 A. S. Ware, P. H. Diamond, B. A. Carreras, J. N. Leboeuf, and D. K. Lee, Phys. Fluids B $4,102(1992)$.

13 A. S. Ware, P. H. Diamond, H. Biglari, B. A. Carreras, L. A. Charlton, J.-N. Leboeuf, A. J. Wootton, (submitted to Phys. Fluids B).

14 L. Chen and C. Z. Cheng, Phys. Fluids 23, 2242 (1980).

15 S. I. Braginskii, in Reviews of Plasma Physics, edited by M. A. Leontovich (Consultants Bureau, New York, 1965), Vol. I, p. 205.

16 J. F. Drake, L. Sparks, and G. Van Hoven, Phys. Fluids 31, 813 (1988). 
17 J. W. Connor, B.. J. Hastie, and J. B. Taylor, Proc. R. Soc. 365, 1 (1979).

18 A. H. Glasser, in Proceedings of the Finite Beta Theory Workshop, Varenna, 1977, edited by B. Coppi and W. Sadowski (U. S. Department of Energy, CONF-7709167, 1977), p. 55.

19 Y. C. Lee and J. W. Van Dam, in Proceedings of the Finite Beta Theory Workshop, Varenna, 1977, edited by B. Coppi and W. Sadowski (U. S. Department of Energy, CONF-7709167, 1977), p. 93.

20 R. B. White, J. Comput. Phys. 31, 409 (1979).

21 D. E. Post, R. V. Jensen, C. B. Tarter, W. H. Grasberger, and W. A. Lokke, At. Data Nucl. Data Tables 20, 397 (1977).

22 A. S. Vrare and P. H. Diamond, private communication, 1991.

23 A. Hasegawa and K. Mima, Phys. Rev. Lett. 39, 205 (1977).

24 B. B. Kadomtsev and O. P. Pogutse, Sov. Phys. JETP 24, 1172 (1967). 
Table I. Typical Edge Parameters

\begin{tabular}{|l||r|r|}
\hline & TFTR & TEXT \\
\hline$n_{e}\left(\mathrm{~cm}^{-3}\right)$ & $8 \times 10^{12}$ & $8 \times 10^{12}$ \\
$T_{e}(\mathrm{eV})$ & 150 & 40 \\
$n_{N}\left(\mathrm{~cm}^{-3}\right)$ & $3 \times 10^{9}$ & $3 \times 10^{10}$ \\
$P_{\text {rad }}\left(\mathrm{W} / \mathrm{cm}^{3}\right)$ & 0.03 & 0.1 \\
$L_{n}(\mathrm{~cm})$ & 10 & 4 \\
$L_{T}(\mathrm{~cm})$ & 5 & 2 \\
$\eta_{e}$ & 2 & 2 \\
$a(\mathrm{~cm})$ & 80 & 27 \\
$R_{0}(\mathrm{~cm})$ & 245 & 100 \\
$B_{0}(\mathrm{~T})$ & 4.5 & 2 \\
$q$ & 4 & 4 \\
$\hat{s}$ & 2 & 2 \\
$\epsilon_{n}$ & .04 & .15 \\
$c_{s}\left(\mathrm{~cm}^{2}\right)$ & $8.5 \times 10^{6}$ & $4.4 \times 10^{6}$ \\
$\nu_{e i}\left(\mathrm{~s}^{-1}\right)$ & $1.9 \times 10^{5}$ & $1.4 \times 10^{6}$ \\
$\rho_{s}\left(\mathrm{~cm}^{-1}\right)$ for $k_{\theta} \rho_{s}=0.1$ & $8.9 \times 10^{-2}$ & $4.6 \times 10^{-2}$ \\
$\omega_{* e}\left(\mathrm{~s}^{-1}\right)$ & $\left(m=10^{4}\right.$ & $1.1 \times 10^{5}$ \\
$\omega_{* e}\left(\mathrm{~s}^{-1}\right)$ for $k_{\theta} \rho_{s}=0.02$ & $1.7 \times 10^{4}$ & $(m=56)$ \\
$\beta_{n}\left(\mathrm{~s}^{-1}\right)$ & $(m=39)$ & $(m=11)$ \\
$\beta_{T}\left(\mathrm{~s}^{-1}\right)$ & 100 & 800 \\
$\gamma_{v}\left(\mathrm{~s}^{-1}\right)$ & -10 & 300 \\
$\gamma_{n}\left(\mathrm{~s}^{-1}\right)$ & 200 & 1000 \\
$\gamma_{T}\left(\mathrm{~s}^{-1}\right)$ & 100 & 1300 \\
\hline & -200 & 1400 \\
\hline
\end{tabular}




\section{Figure Captions}

Fig. 1. Numerical solution of Eq. (24a) for extremely long wavelength $\left(k_{\theta} \rho_{s}=0.02\right)$, with $q=4$, $\epsilon_{n}=0.1$, and $\hat{s}=2$. (a) Effective potential $-Q(\eta)$ in ballooning cocrdinate. (b) Lowest order eigenmode in ballooning coordinate, $\Phi(\eta)$. (c) Lowest order eigenrnode in radial coordinate, $\Phi(z)$.

Fig. 2. Numerical solution of Eq. (24a) for $k_{\theta} \rho_{s}=0.1$, with $q=4, \epsilon_{n}=0.1$, and $\hat{s}=2$. (a) Effective potential $-Q(\eta)$ in ballooning coordinate. (b) Lowest order eigenmode in ballooning coordinate, $\Phi(\eta)$. (c) Lowest order eigenmode in radial coordinate, $\Phi(z)$.

Fig. 3. Spatial dependence of $\tilde{n}$ (solid) and $\tilde{T}$ (dashed) for $\bar{\nu} \ll 1$.

Fig. 4. Spatial dependence of $\tilde{n}$ (solid) and $\tilde{T}$ (dashed) for $\bar{\nu} \gg 1$.

Fig. 5. Typical parameter regimes for toroidal drift waves in TFTR and TEXT (electron response).

Fig. 6. Perturbed potential for eigenmode in Fig. 1.

Fig. 7. Perturbed potential for eigenmode in Fig. 2.

Fig. 8. Comparison of analytically (dashed) and numerically (solid) computed growth rates as (a) $\bar{\nu}$, (b) $\eta_{e}$, (c) $\beta_{n}$, (d) $\beta_{T}$, (e) $\gamma_{v}$, (f) $\gamma_{n}$, and (g) $\gamma_{T}$ are varied, using the parameters in Fig. 1. 


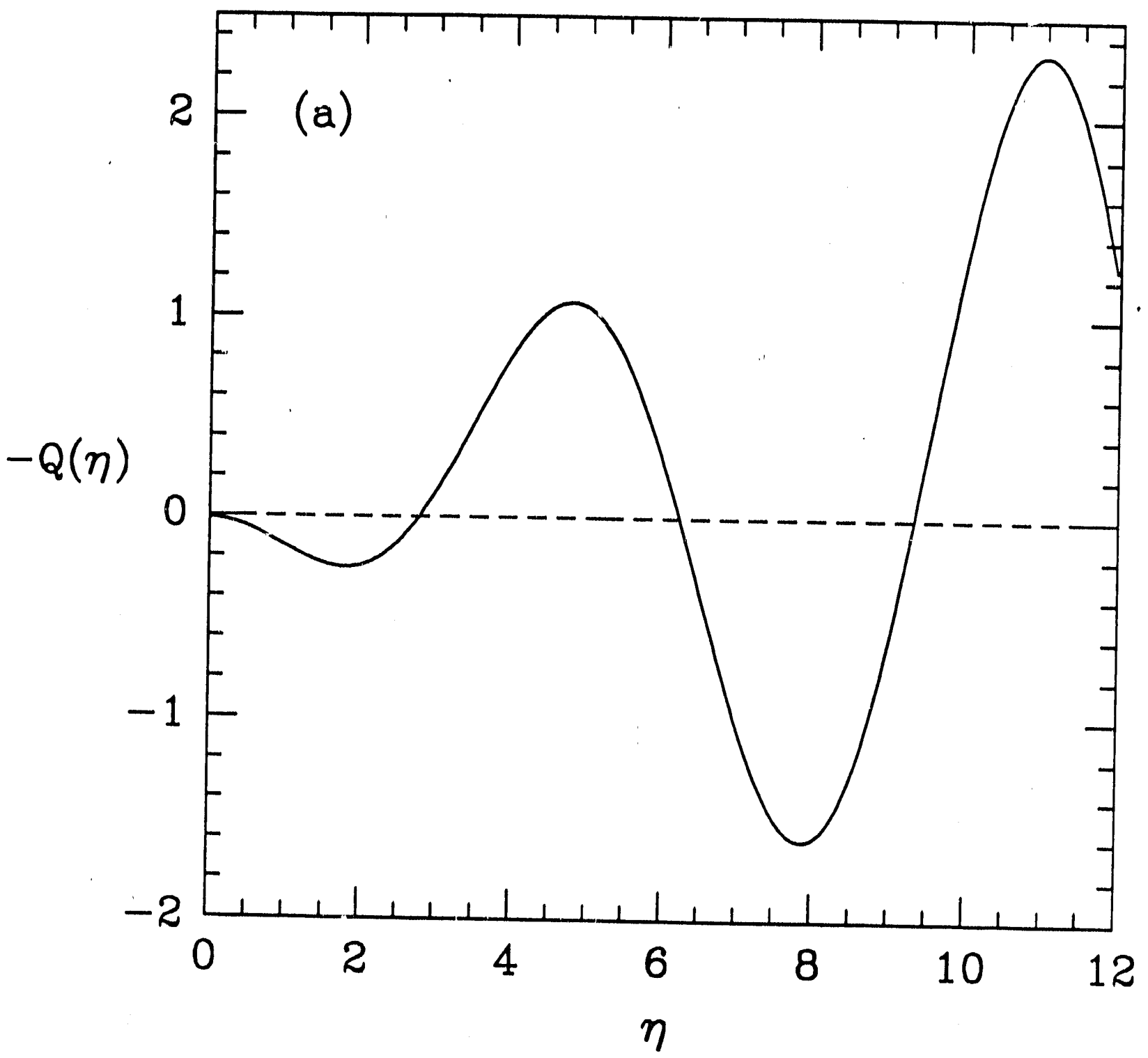

Fig. 1(a). 


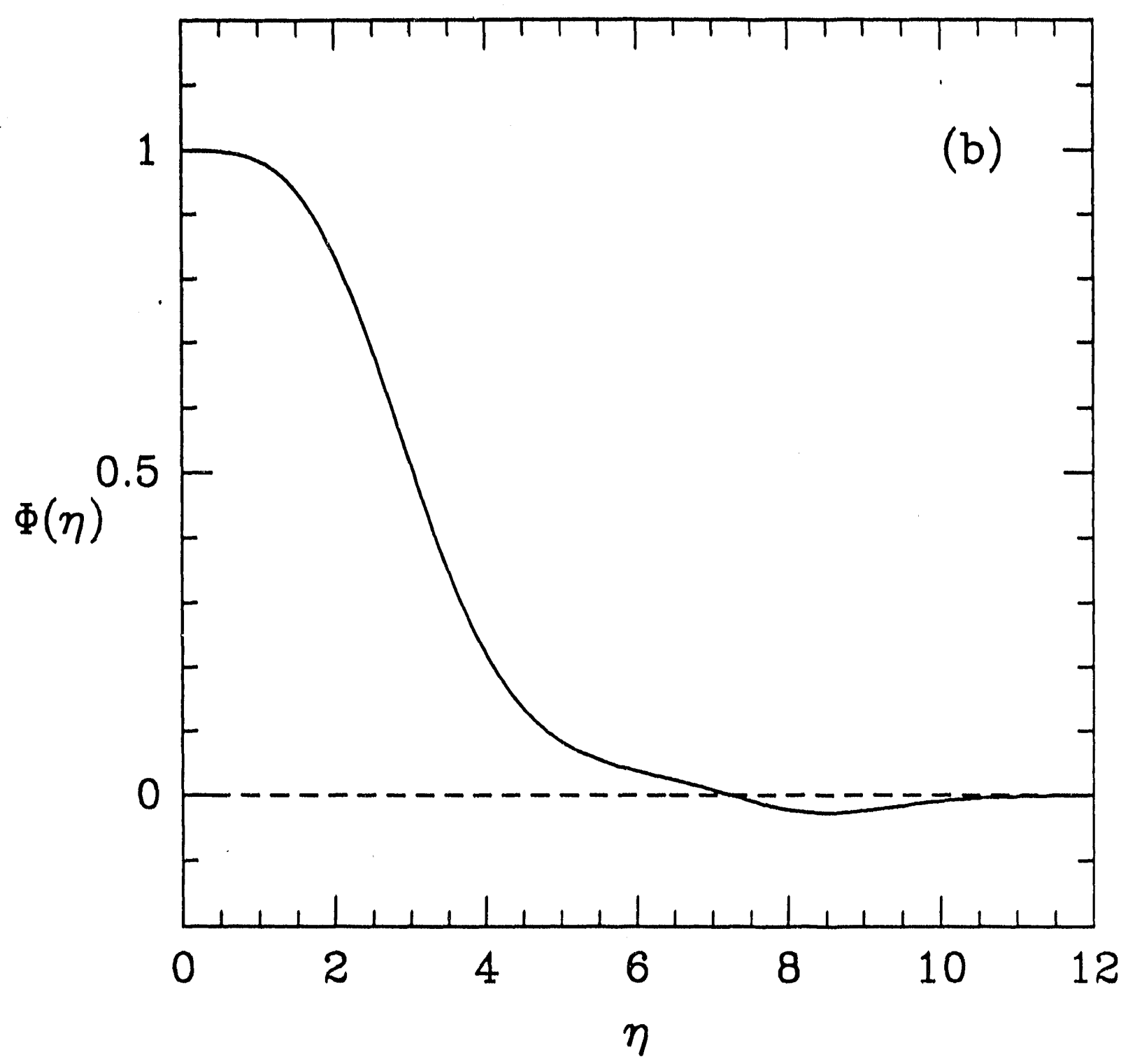

Fig. 1(b). 


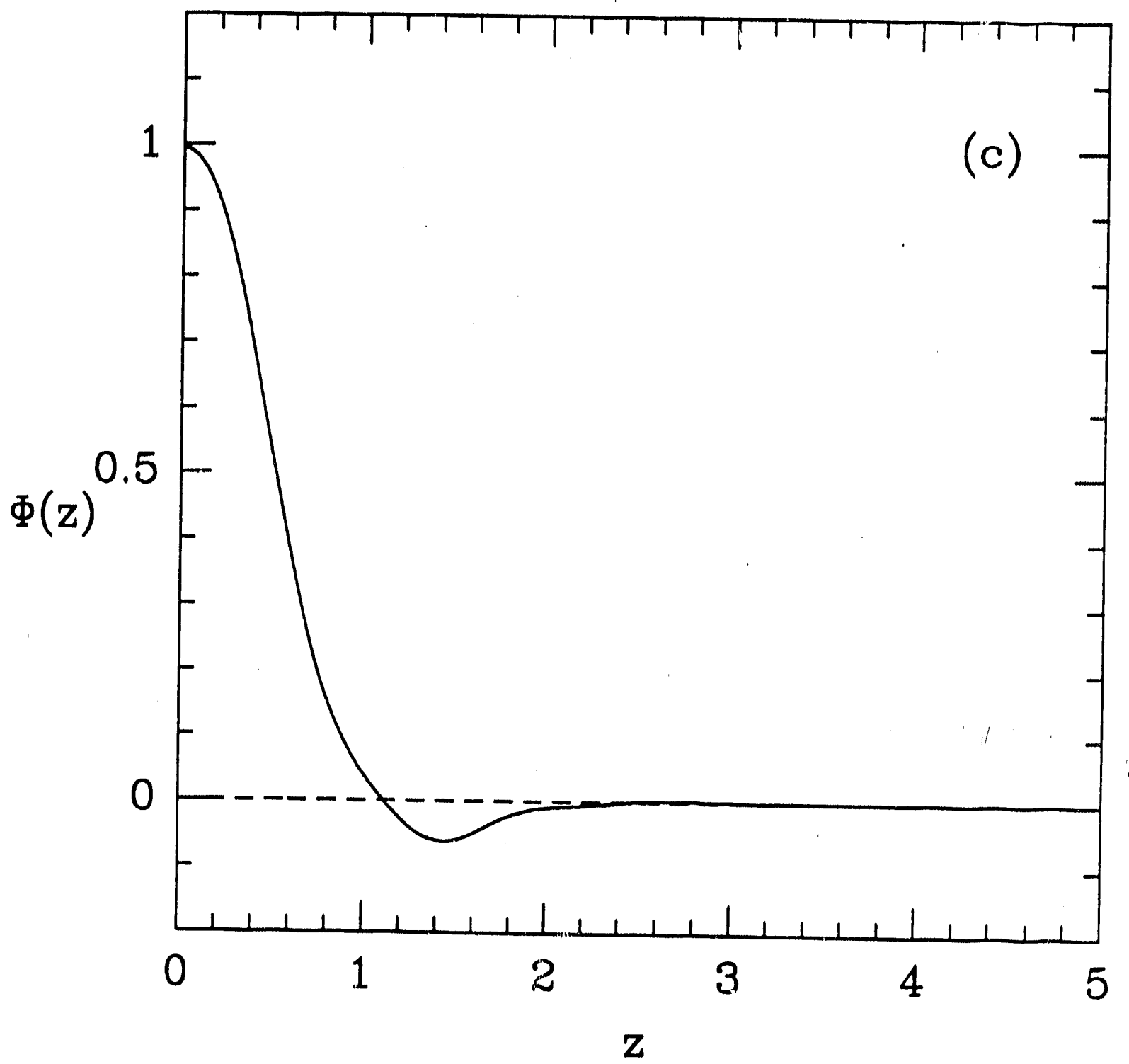

Fig. 1(c). 


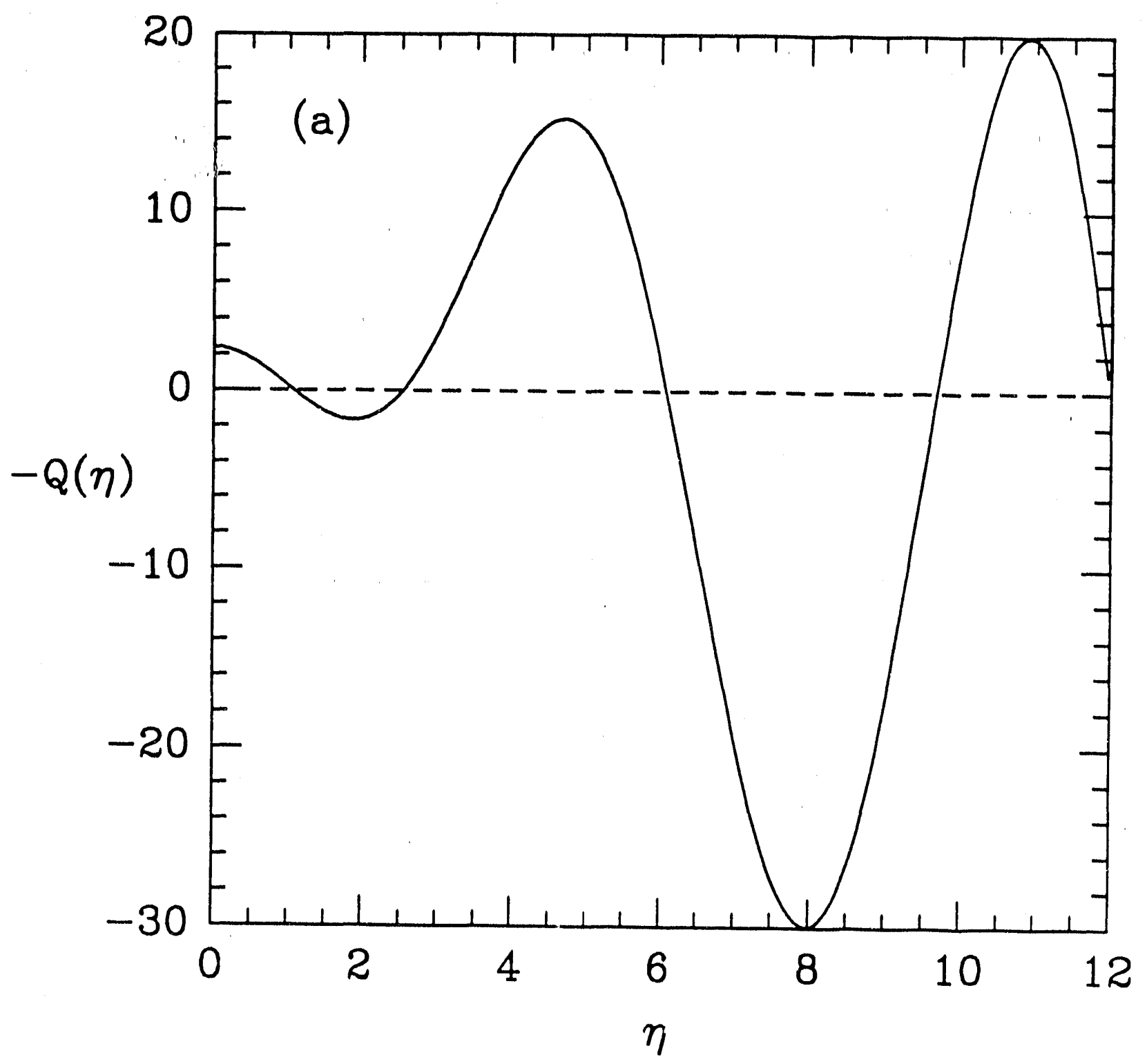

Fig. 2(a). 


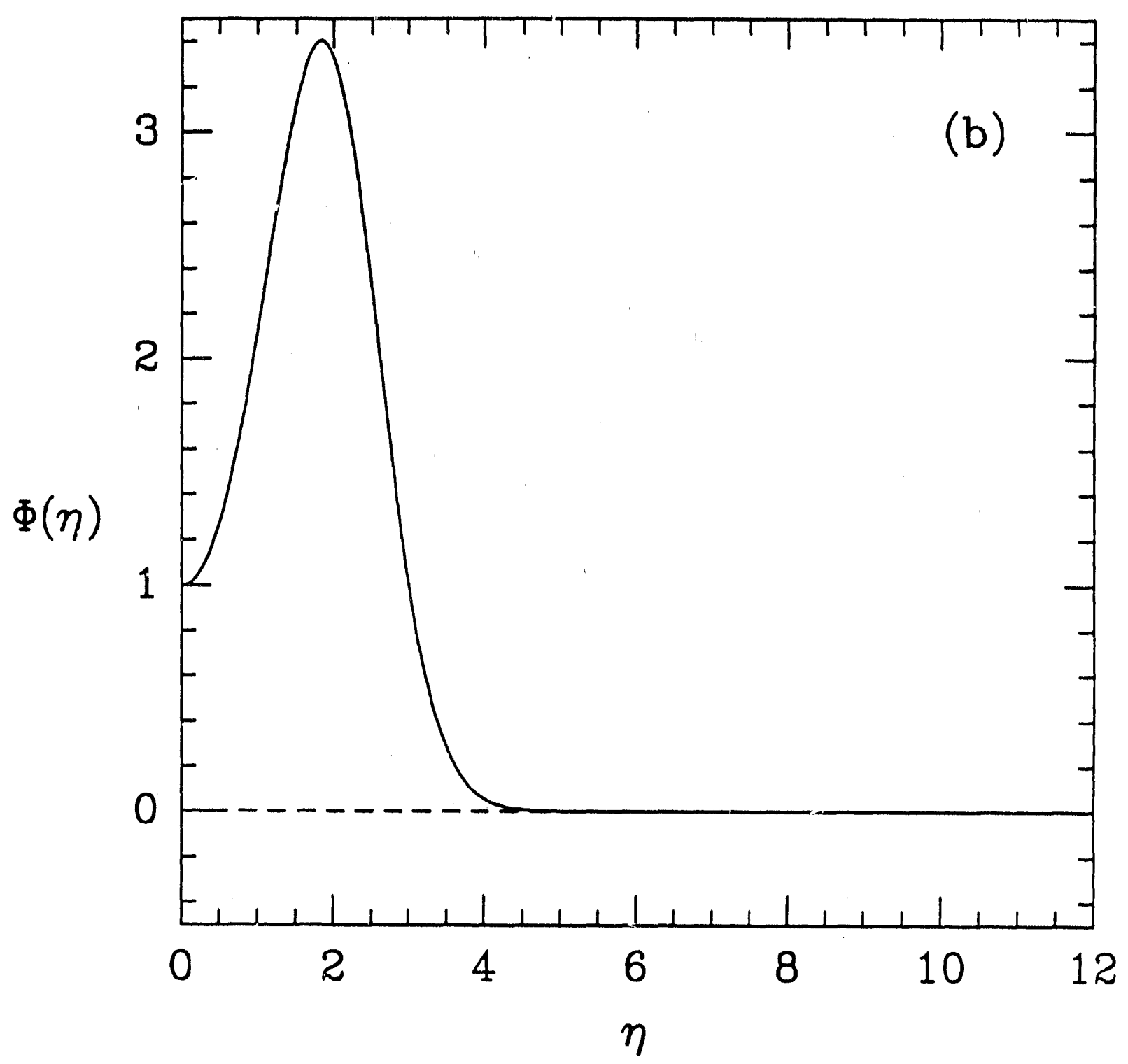

Fig. 2(b). 


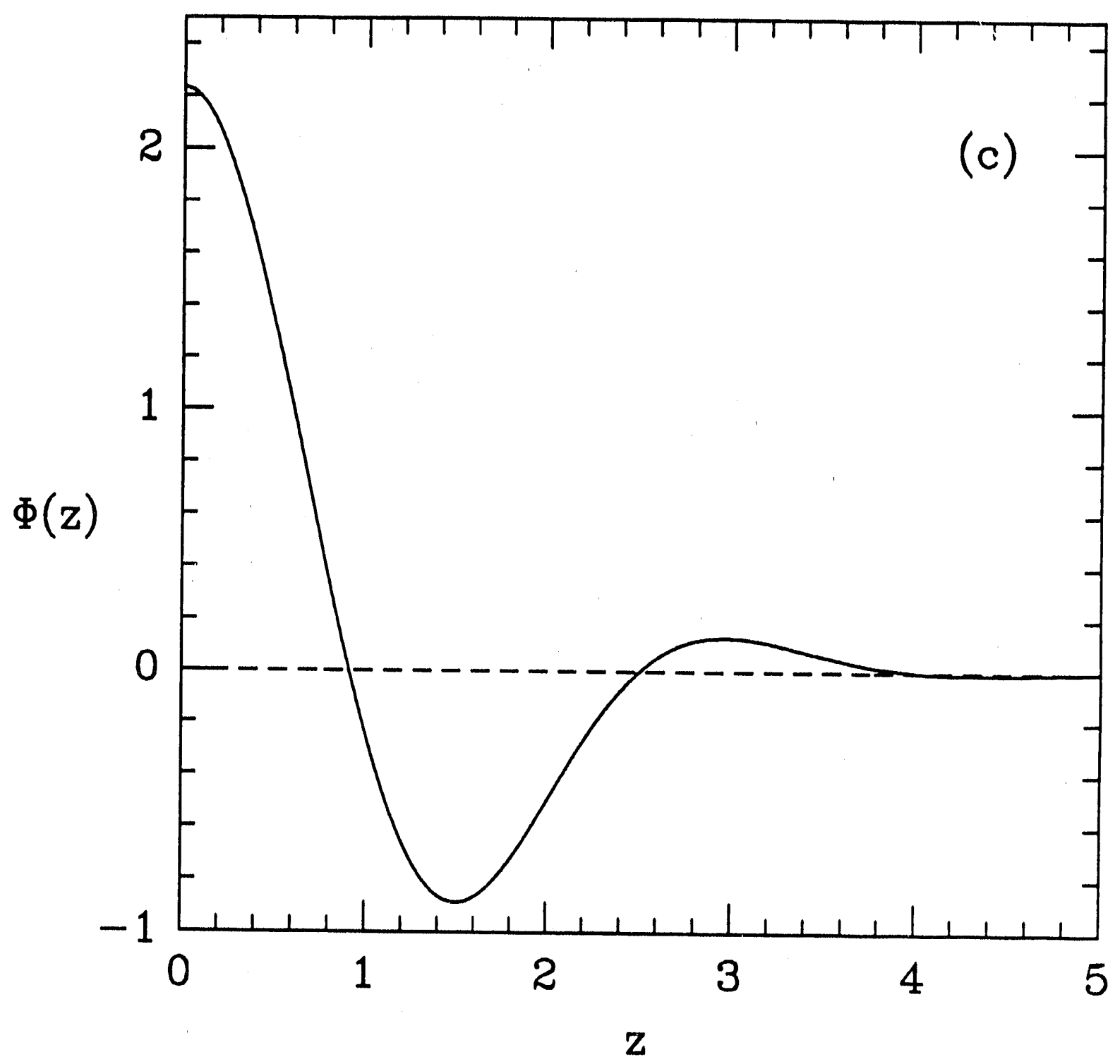

Fig. 2(c). 


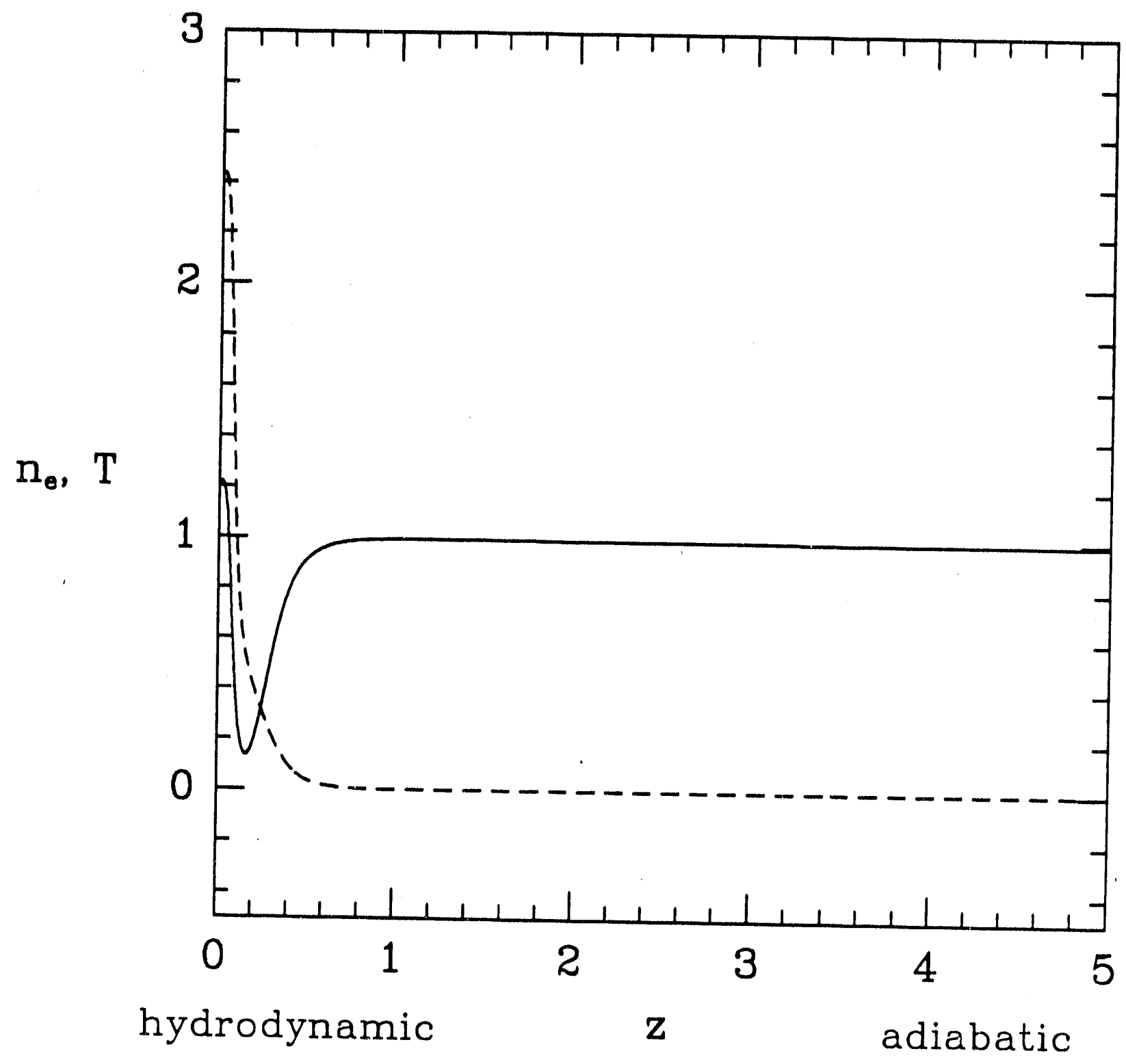

Fig. 3. 


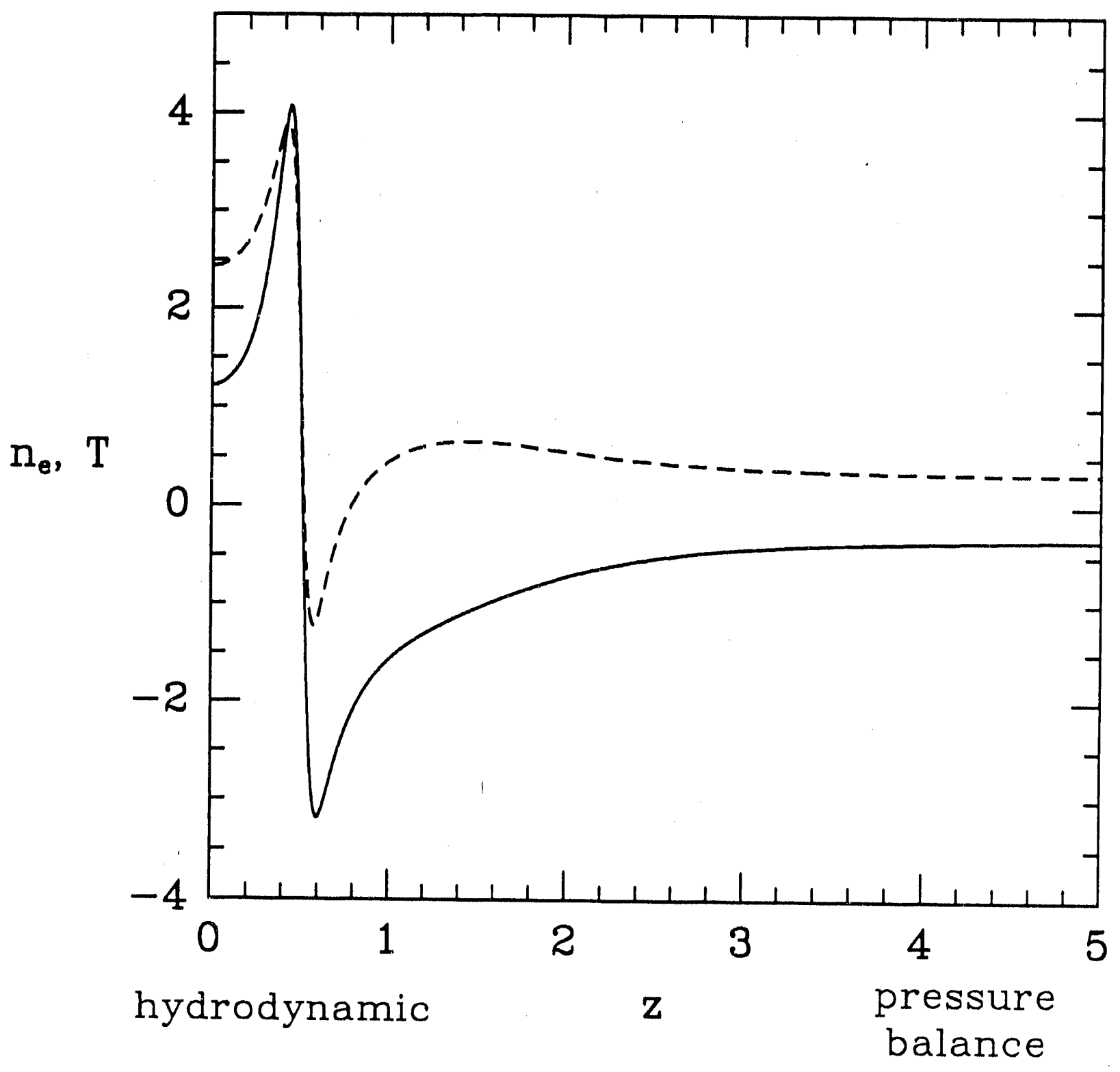

Fig. 4. 


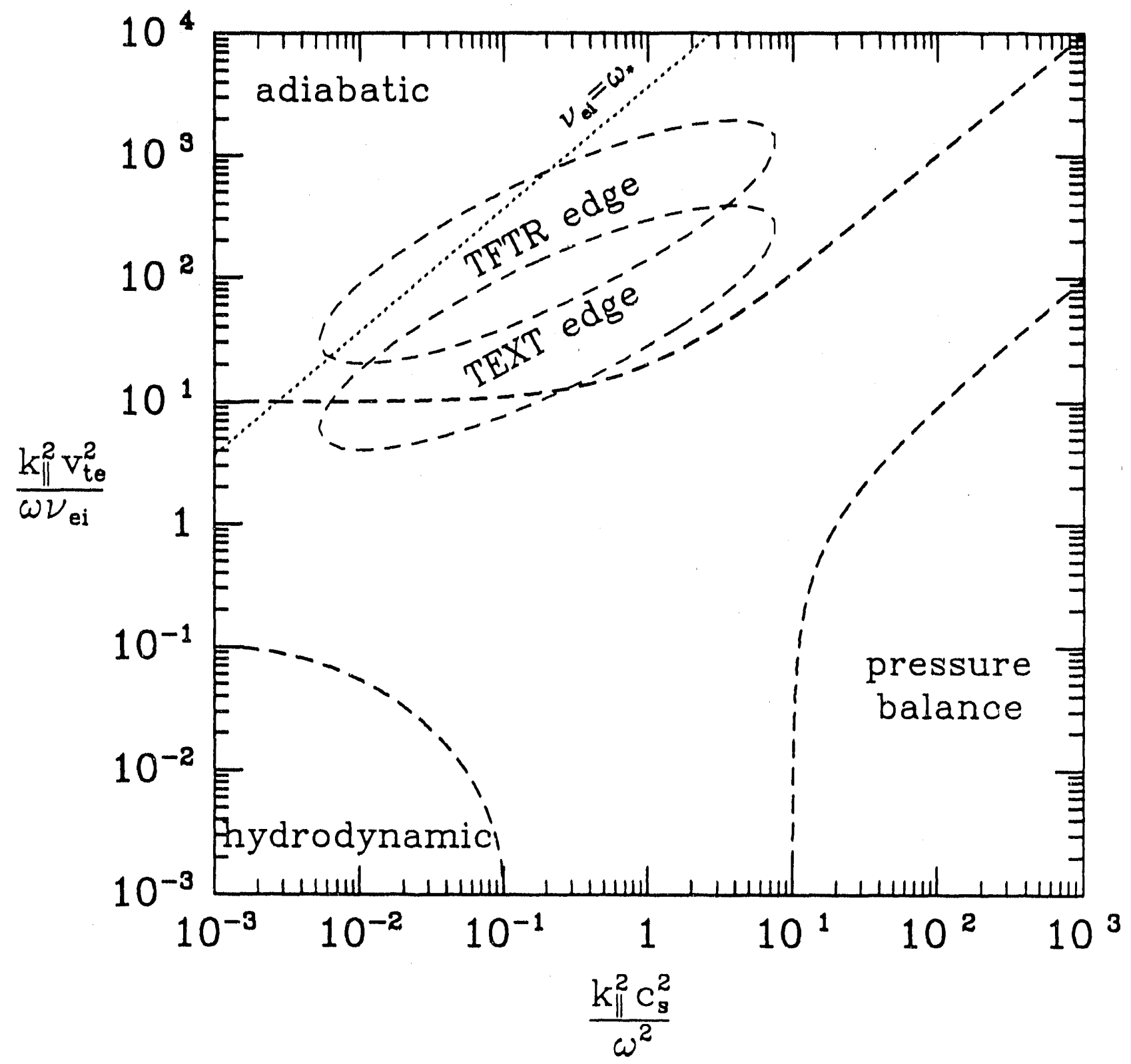

Fig. 5. 


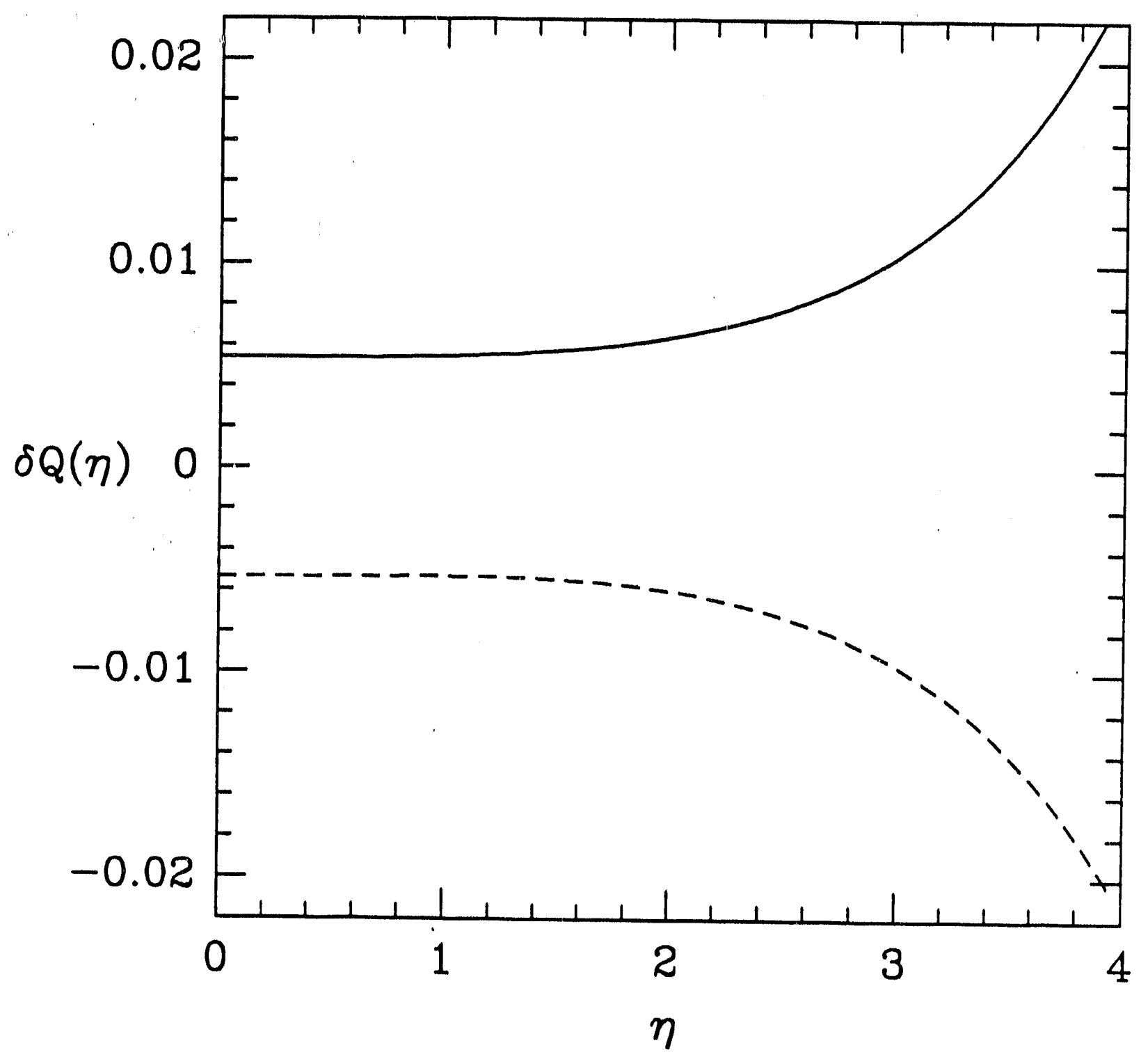

Fig. 6. 


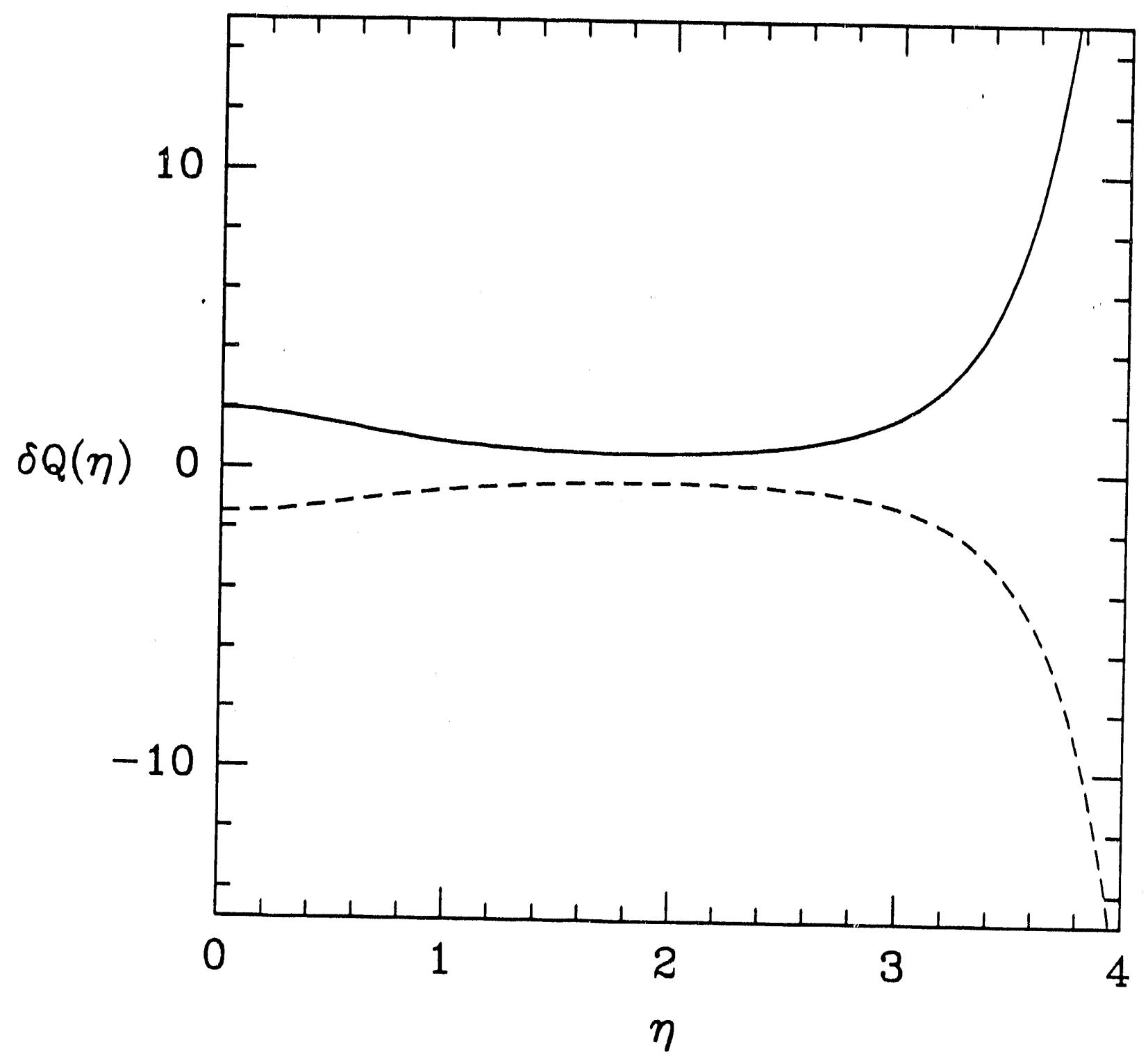

Fig. 7 . 


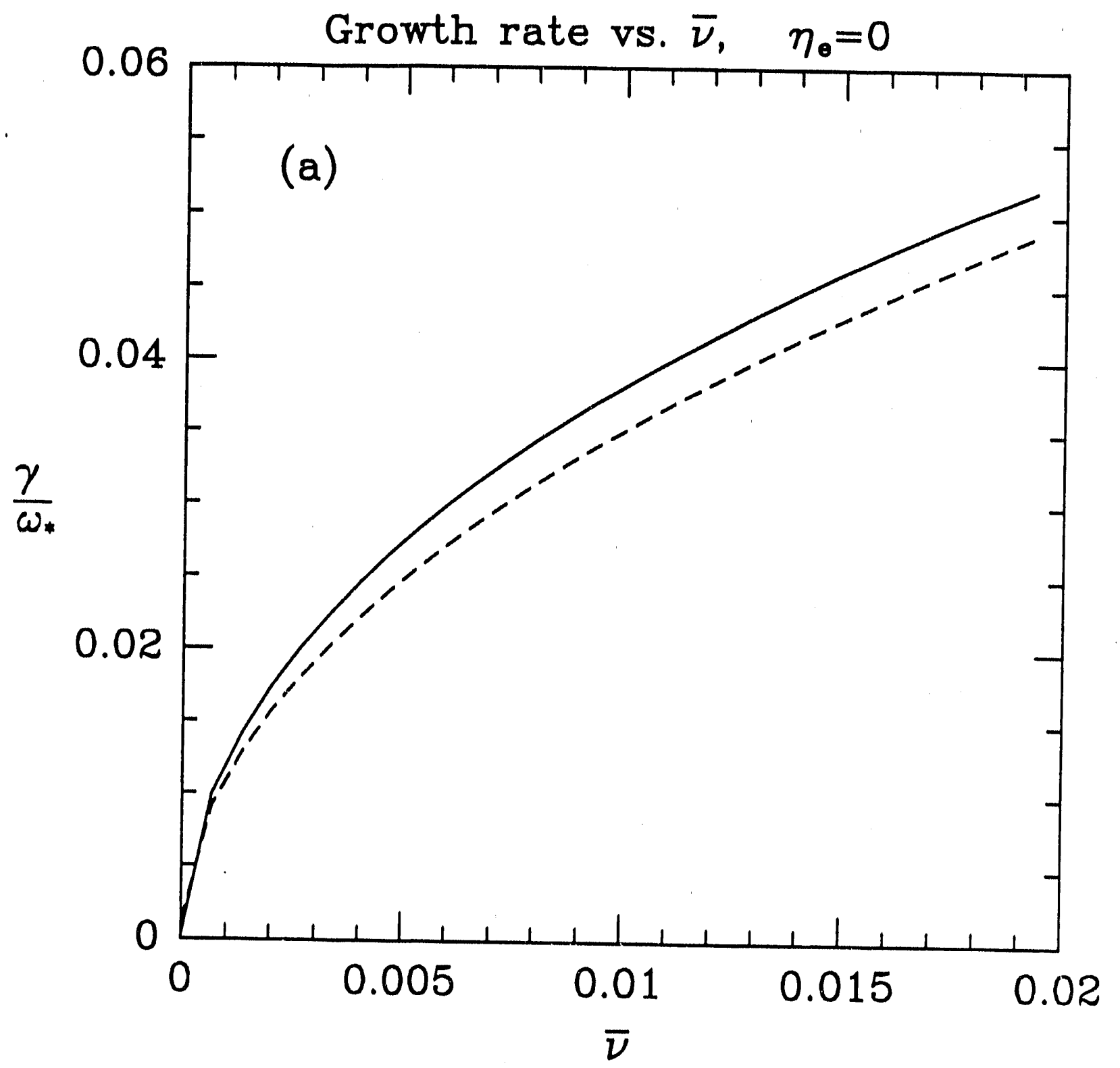

Fig. 8(a). 


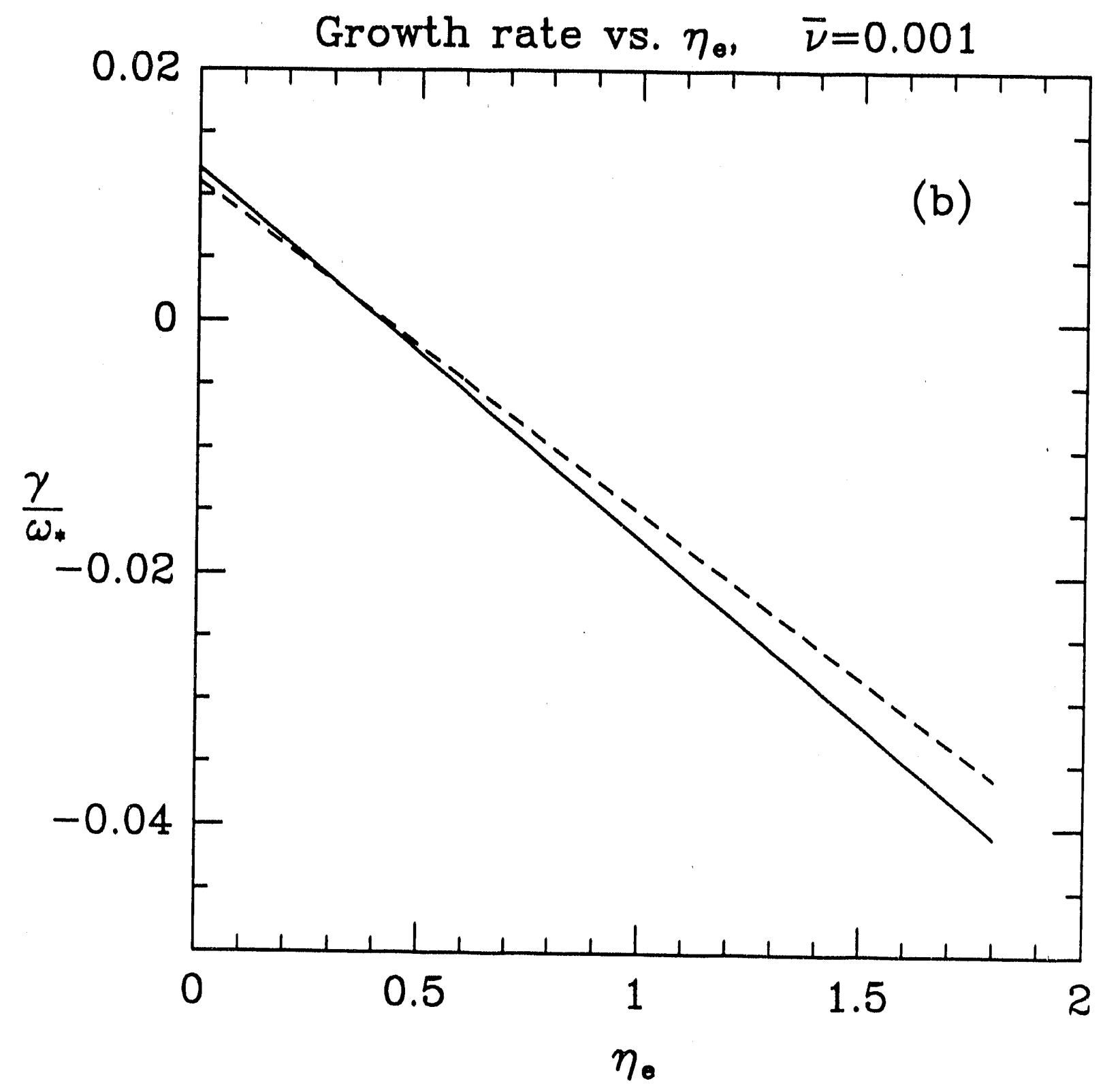

Fig. 8(b). 


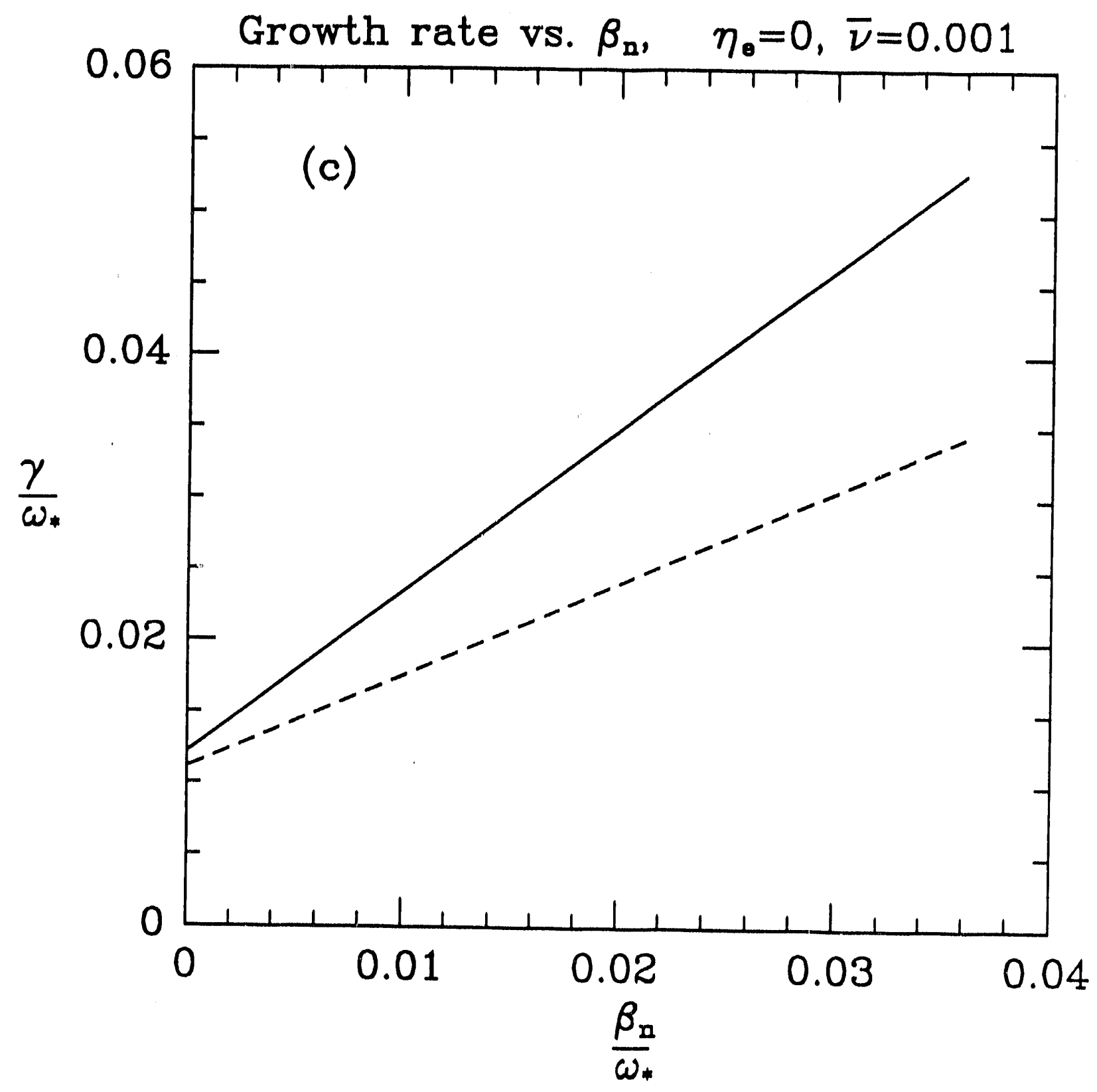

Fig. $8(\mathrm{c})$. 


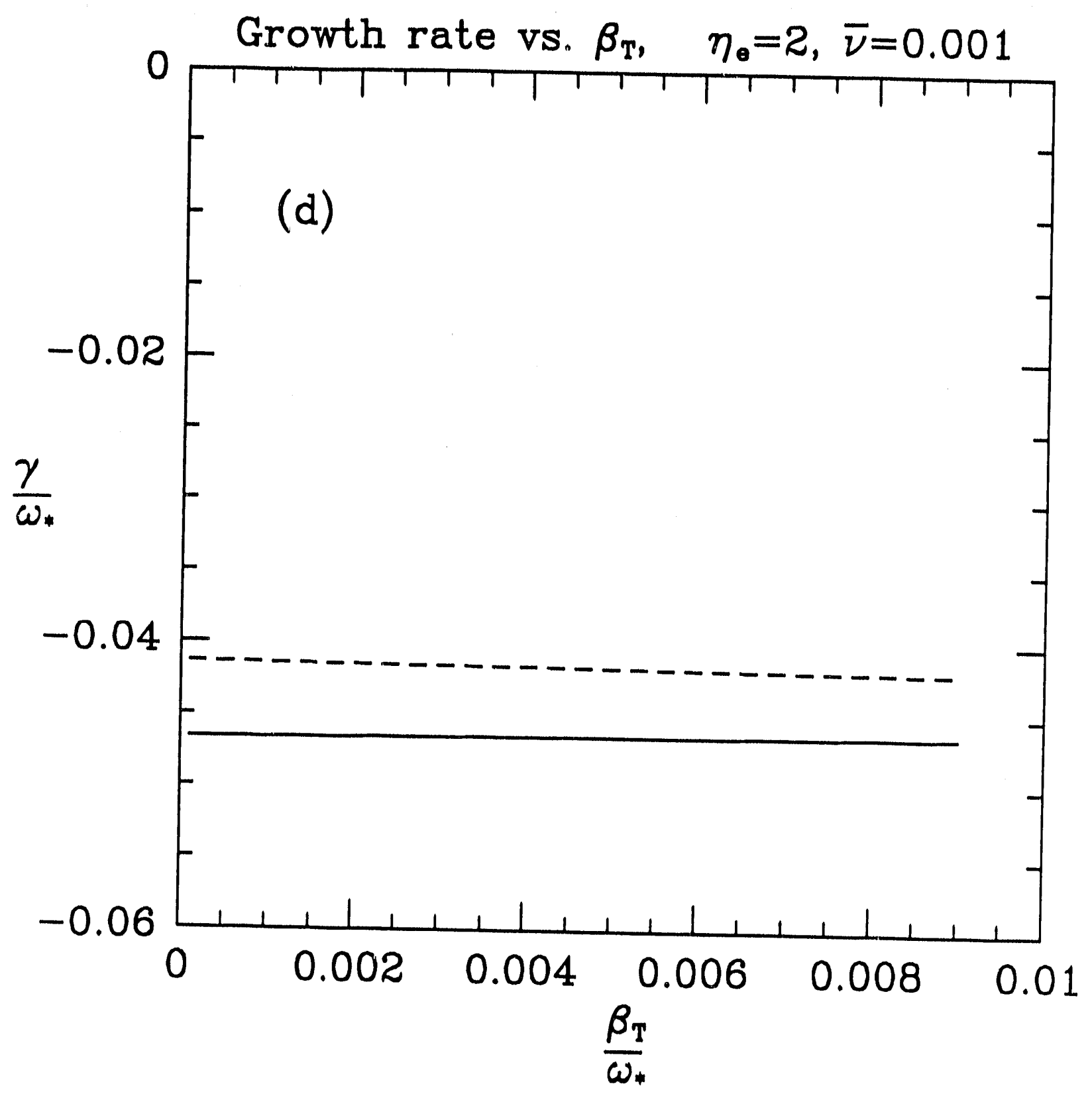

Fig. 8(d). 


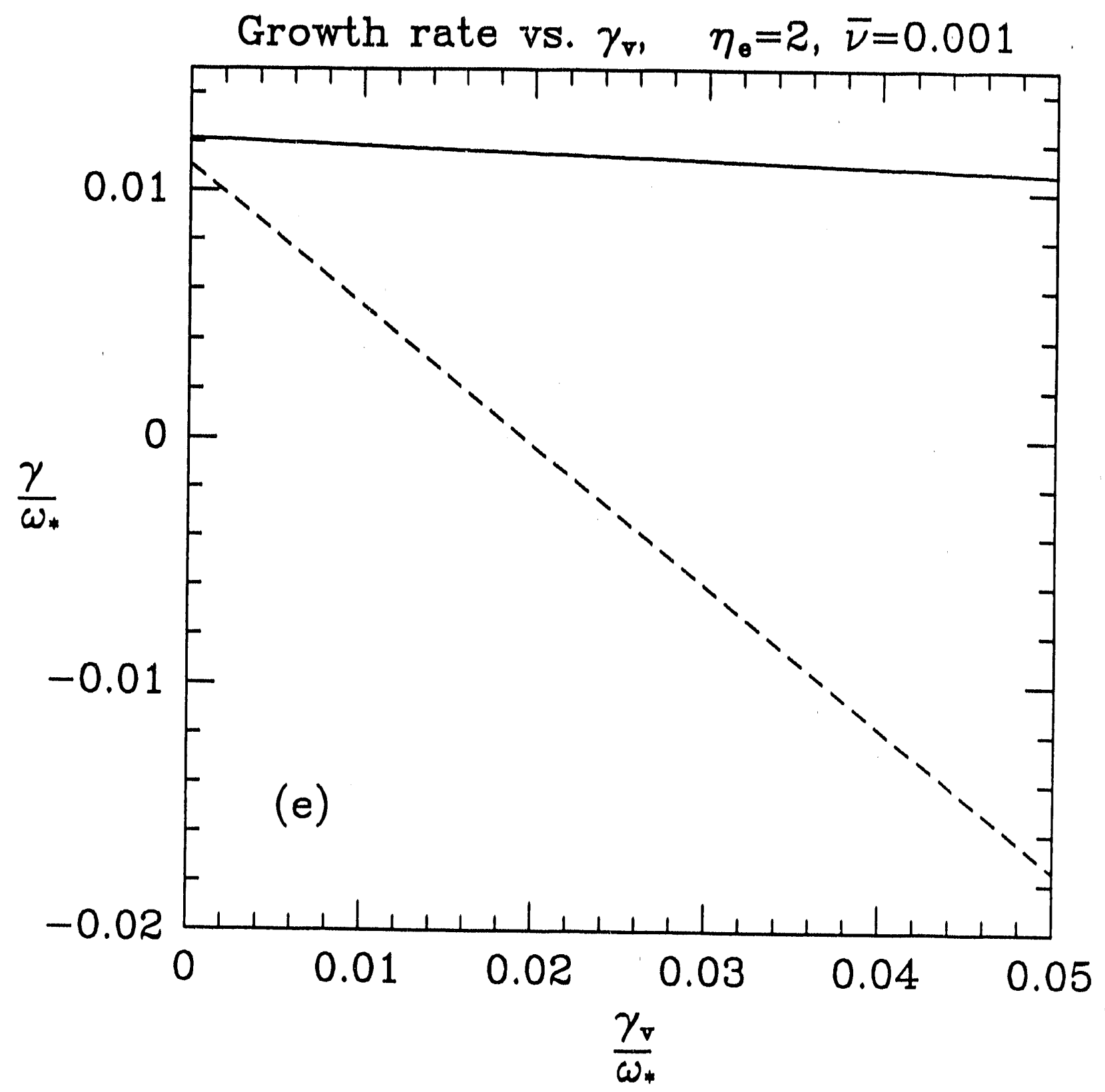

Fig. 8(e), 


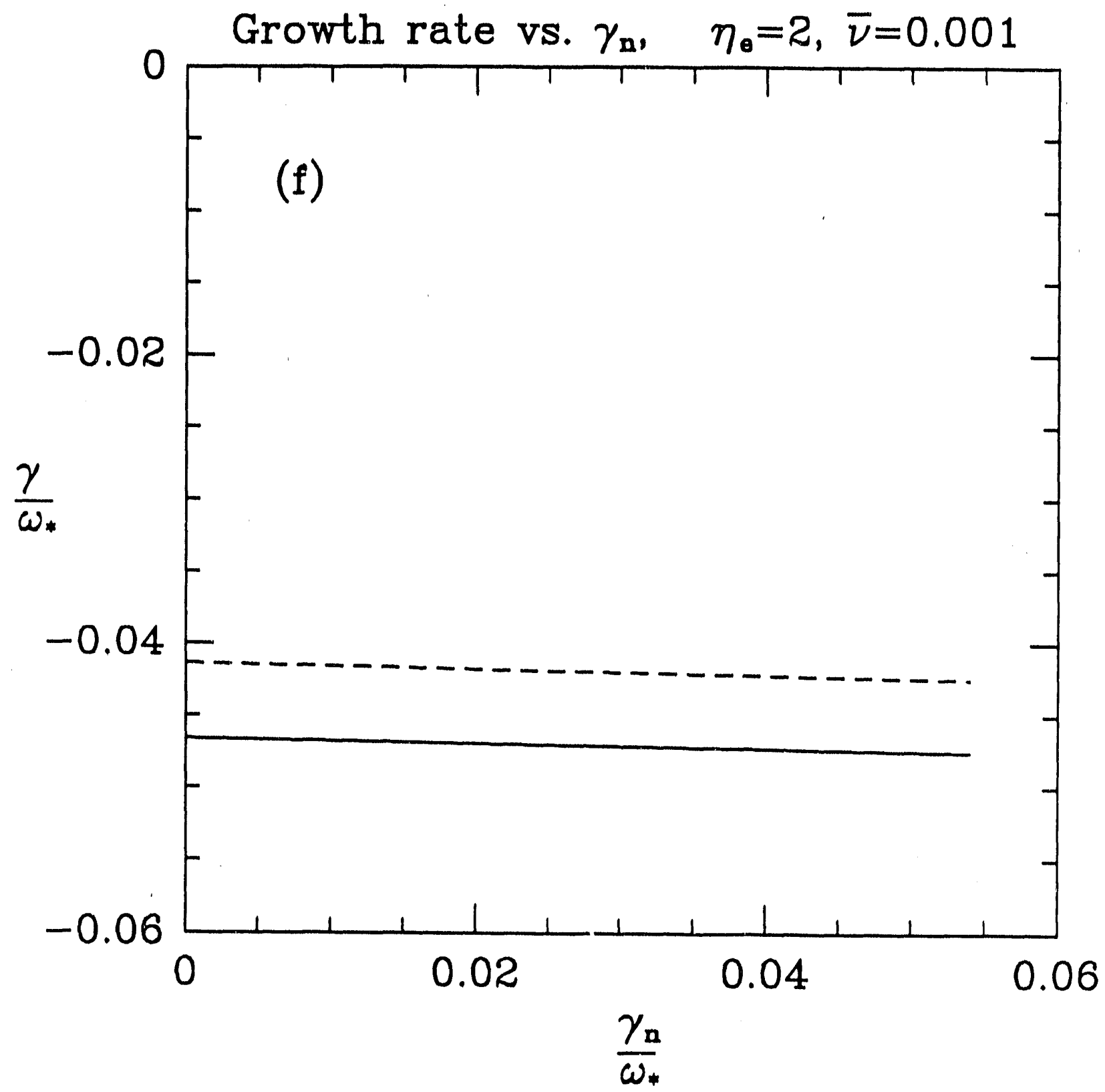

Fig. 8(f). 


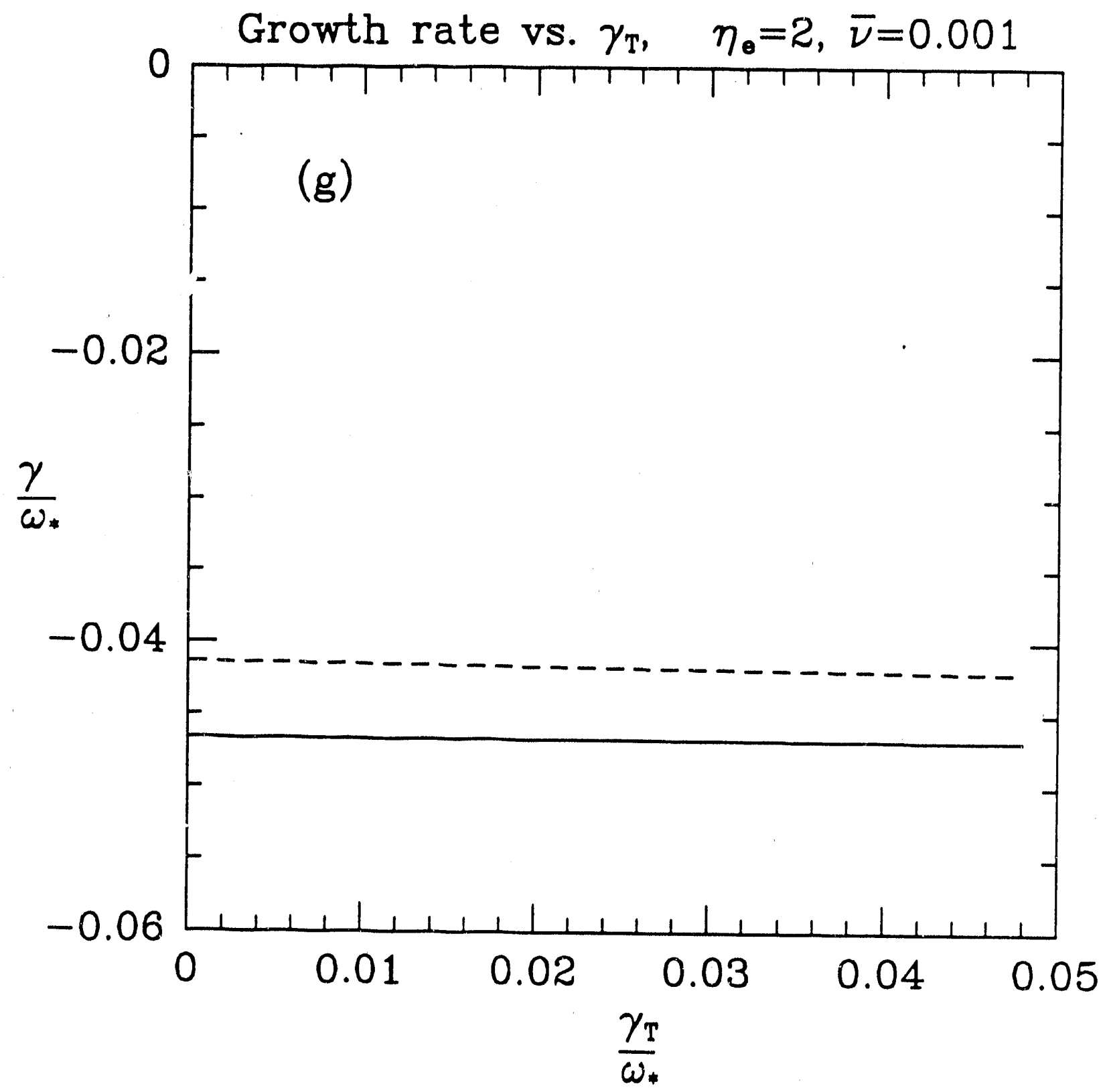

Fig. $8(\mathrm{~g})$. 
Dr. F. Peotoni, Univ. of Wollongong, AUSTRALIA

'Prof. M.H. Brennan, Univ. of Syonoy, AUSTRALIA

Plasma Rosearch Leb., Australien Nat. Univ., AUSTRALIA

Prof. I.R. Jones, Finders Univ, AUSTRALLA

Prot. F. Cep, Inst. for Theoretical Physica, AUSTRIA

Prot. M. Heinclior, Instiut for Theoretische Phyeik, AUSTRIA

Prof. M. Goossens, Atronomisch Inetiuut, BELGIUM

Ecolo Roy do Nituine, Leb. do Phy. Plaemas, BELGIUM

Commicuion-Europeen, DG. XII-Fusion Prog., BELGIUM

Prof. R. Bouciaut, Rikfuniveratbit Gent, BELGIUM

Dr. P.H. Sakenake, Institurio Fivica, BPUZIL

Instituto Naciond Do Prequiteas Espacidie-INPE, BRUZIL

Documents Orices, Alomic Energy of Ceneda LU., CANADA

Dr. M.P. Eectynnetu, MPB Technologies, Inc., CANADA

Dr. H.M. Skaragard, Univ. of Sackatchowen, CANADA

Prot. J. Teichmann, Univ. of Montrid, CANADA

Prol. S.R. Srecenivesen, Univ. of Celogry, CANAOA

Prof. T.W. Johnston, INRS-Energio, CANADA

Dr. R. Botion, Contre canadien de husion manosiquo, CANADA

Dr. C.R. James., Univ. of Nberta, CANADA

Dr. P. Lukle, Komensketho Universzitu, CZECHOSLOVAKIA

The Librarian, Cuthem Leboratory, ENGLAND

Librey, R61, Ruthortord Apploton Laboratory, ENGLAND

Mrs. S.A. Hutchinson, JET Librery, ENGLAND

Dr. S.C. Shama, Univ. of South Pecific, FWII ISLANDS

P. Mathonen, Univ. of Helsinki, FINLAND

Prof. M.N. Buseac, Ecols Potylochnique, FRANCE

C. Moutrat, Lob. de Phyeique des Milioux lonieds, FRANCE

J. Radit, CENCADARACHE - Bat 506, FRANCE

Prot. E. Economou, Univ. of Crow, GREECE

Ms. C. Pinni, Univ. of loamina, GREECE

Dr. T. Mued, Acadermy Bibwogrephic Ser., HONG KONG

Proprint Librery, Hungarian Acaderny of Sci., HUNGARY

Dr. B. DesGupta, Satia inst of Nuctoer Ptysics, INDIA

Dr. P. Kaw, Inst. ker Plaema Research, INDLA

Dr. P. Rocenew, Iered Inst of Technobogy, ISAAEL

Librerien, Intemational Comber for Theo Physics, ITALY

Miss C. Do Palo, Associazione EURATOMENEA, ITALY

Dr. G. Groseo, lesituto di Fieica ded Plasma, ITALY

Prof. G. Rustenoni, lstitur Gas lonizzati Del Cnr, ITALY

Dr. H. Yemeto, Toutiba Res a Dowel Center, JAPAN
Prof. I. Kewakemi, Hroshima Univ., JAPAN

Prof. K. Nishikawa, Hiroshima Univ., JAPAN

Director, Jepen Alomic Energy Rosearch Inst., JAPAN

Prot. S. Itoh, Kyushu Univ., JAPAN

Plesearch Into. Cr., National Instit. for Fusion Science, JAPAN

Prof. S. Tenaka, Kyoob Univ., JAPAN

Librery, Kyoto Univ., JAPAN

Prol. N. Inow, Univ. of Tokyo, LAPAN

Secretary, Pleme Section, Eloctrotechnical Lab., JAPAN

S. Mori, Technical Advieor, MAEPI, MAPAN

Dr. O. Muend, Kumamoso mat, of Technology, JAPAN

J. Hyron-Sook, Korea Alomic Enorgy Rosearch Inst, KOAEA

D.I. Choi, The Koree Adv. Inst of Sai. \& Tech., KOREA

Prof. B.S. Liby, Univ, of Weikab, NEW ZEALAND

Inat of Phyrics, Chinese Acad Sa PEOPLE'S REP. OF CHINA

Librery, Inst of Plaema Phyeics, PEOPLE'S REP. OF CHINA

Tainghua Univ. Librey, PEOPLE'S REPUBLIC OF CHINA

Z. U, S.W. Inst Physice, PEOPLE'S REPUBUC OF CHINA

Prof. J.A.C. Cabre, Instituto Superior Tecnico, PORTUGAL

Dr. O. Porrus, A.I CUZA Univ., ROMANIA

Dr. J. do Villiers, Fusion Studios, AEC, S. AFPICA

Prof. M.A. Hewborg. Univ. of Natal, S. AFRICA

Prof. D.E. Kim, Poheng inat. of Sd. \& Tech., SO. KOREA

Prof. C.I.E.M.A.T, Fusion Division Library, SPAIN

Dr. L. Siomo, Univ. of UMEA, SWEDEN

Librery, Royd Inet of Tectnology, SWEDEN

Prot. H. Whelmeon, Chalmore Univ. of Tech., SWEDEN

Contre Phys. Des Phasmas, Ecolo Potytech, SWITZERLAND

Bibliotheak, Inat. Voor Plasma-Fysica, THE NETHERLANDS

Asst Prot. Dr. S. Cakir, Middo East Tech. Univ., TURKEY

Dr. V.A. Gulthikh, Sai. Ree. Inst Electrophys.I Apparatus, USSR

Dr. D.D. Ayutov, Sibarian Branch of Acadomy of Sa., USSR

Dr. G.A. Eliseov, I.V. Kurchator Inst, USSR

Librurian. Tho Ukr.SSA Academy of Scionces, USSA

Dr. LM. Kovizhnykh, Inst. of Generd Physics, USSR

Kentorectungeaniege GmbH, Zentrabibliothek, W. GERMANY

Bibliothok, Inst. FOr Plasmatorechung, W. GERMANY

Prot. K. Schinder, Rutr-Universitu Bochum, W. GERMANY

Or. F. Weoner, (ASOEX), Max-Planck-Institut, W. GERMANY

Litrerien, Max-Planck-Instiut, W. GERMANY

Prof. R.K. Jenov, Inat of Phycica, YUGOSLAVIA 

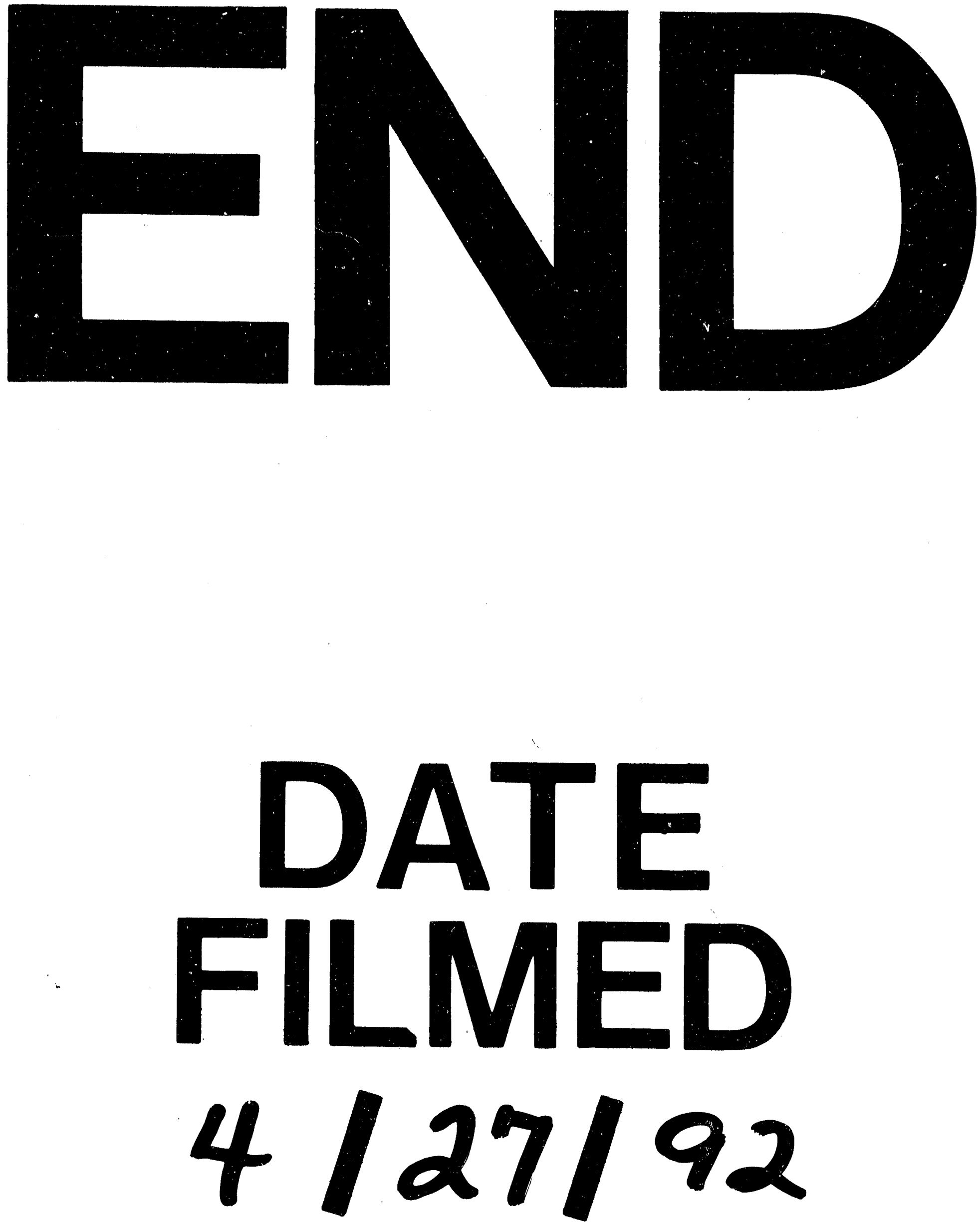
